\title{
GRADE SCHEMES AND GRADE FUNCTIONS
}

BY

\author{
STEPHEN MCADAM
}

\begin{abstract}
In recent years, two concepts similar to $R$-sequences have appeared, essential sequences and asymptotic sequences. This work explores the general nature of such sequences.
\end{abstract}

Introduction. In the last three years, two concepts similar to that of $R$-sequences have appeared, asymptotic sequences and essential sequences. Motivated by these examples, this paper will study the general nature of such sequences, which we will refer to as grade sequences. We will generally restrict our attention to a fixed Noetherian ring $R$ and all of its localizations. For any ideal $I_{S}$ in any localization $R_{S}$, we will assign to $I_{S}$ a finite subset of $A\left(I_{S}\right)$ of $\operatorname{spec} R_{S}$, the function $A$ having certain nice properties to be specified in the next section. Foremost among these nice properties is the following. Suppose $x_{1}, \ldots, x_{n}$ are in $I_{S}$ and $x_{1} \notin A\left(O R_{S}\right)$, and for $i=1, \ldots, n-1, x_{i+1} \notin \bigcup\left\{P \in A\left(\left(x_{1}, \ldots, x_{i}\right) R_{S}\right)\right.$, and also suppose there is a prime $P_{S} \in A\left(\left(x_{1}, \ldots, x_{n}\right) R_{S}\right)$ with $I_{S} \subseteq P_{S}$. Now suppose $y_{1}, \ldots, y_{m}$ in $I_{S}$ behaves similarly. We will then insist that $n=m$. If so, $A$ will be called a grade scheme on $R$, and to $A$ we will associate a so-called grade function $f$ defined by $f\left(I_{S}\right)=n, n$ as above. The most well-known instance of such an $A$ and $f$ is, of course, $A\left(I_{S}\right)=$ $\operatorname{Ass}\left(R_{S} / I_{S}\right)$, and $f\left(I_{S}\right)$ is the grade of $I_{S}$. As we will use the term 'grade' more generally, this famous special case will herein be called classical grade. In the next section, we will also give essential grade and asymptotic grade as examples of what we are studying. As mentioned earlier, we will usually just be looking at a grade scheme or grade function on a fixed ring $R$, and we will often custom build a grade function to exist on a given ring $R$. Certain grade functions, primarily classical, essential, and asymptotic grade, as well as the height function, automatically exist on all Noetherian rings, and we will refer to these as natural grade functions. The two-fold purpose of this paper is to study grade functions in the abstract, as well as to investigate which properties of the known natural grade functions are abstract properties, as opposed to being specific to the example at hand.

In $§ 1$, we make precise our definitions, and give the various natural examples. An important point made in our examples is that two different grade schemes can give rise to the same grade function. We give some useful technical lemmas concerning

Received by the editors February 10, 1984.

1980 Mathematics Subject Classification. Primary 13E99; Secondary 13B99.

Key words and phrases. Asymptotic grade, asymptotic prime divisor, classical grade, essential grade, essential prime divisor, grade, grade function, grade scheme, modulated, Noetherian ring, prime divisor, $R$-sequence. 
that fact. $\$ 2$ is the heart of the paper. It contains a characterization of grade functions which is used repeatedly in the rest of the paper, both for proving results and giving concrete examples. Starting with a grade function $f$ on a Noetherian ring $R$, we also give a method of constructing a grade scheme $A_{f}$ for $f$, called the canonical grade scheme for $f$. This canonical grade scheme is easy to work with, and plays a central role in what follows. In $\S 3$ we see how a grade function on $R$ induces grade functions on certain homomorphic images of $R$, and conversely how grade functions on certain collections of homomorphic images of $R$ induce a grade function on $R$. $\$ 4$ studies passing between $R$ and certain extension rings of $R$, notably faithfully flat extensions. In $\S 5$, we use the results of $\$ \S 3$ and 4 to attempt to find a new natural grade function. We develop a concept which gives a grade function on any local ring, and we suggest how it might be extended to all Noetherian rings. Next, let $f$ be any of classical, essential, or asymptotic grade on a local ring $(R, M)$. It is known that if $I$ is an ideal and if $a \in M$, then $f(I, a) \leqslant f(I)$ +1 . In $\S 6$ we study arbitrary grade functions on $(R, M)$ which have this property, and show that if $f$ is one such and $f(M)=$ height $M$, then $f(I)=$ height $I$ for all ideals $I$. We also study the abstract version of the unmixedness theorem, which incorporates the known cases for classical, essential, and asymptotic grade. In $§ 7$, we consider grade sequences over ideal, and given an easy proof of a strong generalization of an inequality known to hold for essential and asymptotic cograde.

1. Definitions and examples. Throughout this paper, $R$ will be a Noetherian ring and $S$ will be an arbitrarily multiplicatively closed subset of $R$ with $0 \notin S$. Thus $R_{S}$ will represent an arbitrary localization of $R$. $I_{S}$ will denote a (proper) ideal of $R_{S}$ (here $I$ is an ideal of $R$ with $I \cap S=\varnothing)$. We will be interested in a function $A\left(I_{S}\right)$ with $A\left(I_{S}\right) \subseteq \operatorname{spec} R_{S}$. (More formally, we should say $A$ is a function defined on the set $\left\{I_{S} \mid I_{S}\right.$ is some ideal in some localization $R_{S}$ of $\left.R\right\}$. However, for the most part we will eschew such formality.)

Definition. The function $A$ mentioned above is called a proto-grade scheme on $R$ if (i) $A\left(I_{S}\right)$ is a finite nonempty subset of spec $R_{S}$, (ii) $P_{S} \in A\left(I_{S}\right)$ implies $I_{S} \subseteq P_{S}$ and (iii) if $P \in \operatorname{spec} R$ and $P \cap S=\varnothing$, then $P \in A(I)$ if and only if $P_{S} \in A\left(I_{S}\right)$.

DEFinition. Let $A$ be a proto-grade scheme. The sequence of elements $x_{1}, \ldots, x_{n}$ in $R_{S}$ is called an avoiding sequence for $A$ if (i) $\left(x_{1}, \ldots, x_{n}\right) R_{S} \neq R_{S}$ and (ii) for $i=1, \ldots, n, x_{i} \notin \bigcup\left\{P \in A\left(\left(x_{1}, \ldots, x_{i-1}\right) R_{S}\right)\right.$.

REMARK. We will often give proofs which induct on the length of some sequence of elements $x_{1}, \ldots, x_{n}$. For these proofs, it will be convenient to agree that when $n=0$, the (empty) sequence is automatically an avoiding sequence for $A$, and the ideal it generates is the zero ideal. In particular, in the previous definition, when $i=1$ we have $\bigcup\{P \in A(0)\}$.

LeMma 1.1. Let $A$ be a proto-grade scheme on $R$.

(i) If $P_{S}$ is a prime minimal over $I_{S}$, then $P_{S} \in A\left(I_{S}\right)$.

(ii) If $x_{1}, \ldots, x_{n}$ is an avoiding sequence for $A$ from $R_{S}$, then height $\left(x_{1}, \ldots, x_{n}\right) R_{S}=$ $n$. 
Proof. (i) As $I_{P}$ is a proper ideal of $R_{P}$, by definition, $A\left(I_{P}\right)$ is nonempty. As every prime in $A\left(I_{P}\right)$ must contain $I_{P}$, we see that $A\left(I_{P}\right)=\left\{P_{P}\right\}$. Thus $P \in A(I)$, and so $P_{S} \in A\left(I_{S}\right)$.

(ii) This is easy by (i) and the principal ideal theorem.

Lemma 1.1 shows that if one builds an avoiding sequence for $A$ by successively adding elements on to the end, the process must stop. This motivates our next definition.

Definition. Let $A$ be a proto-grade scheme on $R$, and let the elements $x_{1}, \ldots, x_{n}$ in $I_{S}$ be an avoiding sequence for $A$. Suppose that for every $x \in I_{S}, x_{1}, \ldots, x_{n}, x$ is not an avoiding sequence for $A$. Then $x_{1}, \ldots, x_{n}$ will be called a maximal avoiding sequence for $A$ in $I_{S}$.

Definition. Let $A$ be a proto-grade scheme on $R$. Suppose for any $I_{S}$, all maximal avoiding sequences for $A$ in $I_{S}$ have the same length. Then $A$ will be called a grade scheme on $R$, and avoiding sequences for $A$ will be called $A$-sequences.

Definition. Let $A$ be a grade scheme on $R$. Let $f\left(I_{S}\right)$ be the length of a maximal $A$-sequence in $I_{S}$. The function $f$ will be called the grade function of $A$. (It is only defined on proper ideals.)

Definition. A function $f$ defined on the set of ideals $I_{S}$ will be called a grade function on $R$, if it is the grade function of some grade scheme on $R$.

LEMMA 1.2. Let $A$ be a grade scheme on $R$ with grade function $f$.

(i) If $I_{S} \subseteq J_{S}$ are ideals of $R_{S}, f\left(I_{S}\right) \leqslant f\left(J_{S}\right)$.

(ii) If $x_{1}, \ldots, x_{n}$ is an $A$-sequence in $R$, and if $\left(x_{1}, \ldots, x_{n}\right) R_{S} \neq R_{S}$, then $x_{1}, \ldots, x_{n}$ is an A-sequence in $R_{S}$. (Here, we should refer to the images of $x_{1}, \ldots, x_{n}$ in $R_{S}$, but this is another formality we will ignore.)

(iii) If $I_{S} \neq R_{S}$, then $f(I) \leqslant f\left(I_{S}\right)$.

(iv) Let $x_{1}, \ldots, x_{n}$ be an $A$-sequence in $R$, and let $\left(x_{1}, \ldots, x_{n}\right) R \subseteq P \in \operatorname{spec} R$. Then $P \in A\left(\left(x_{1}, \ldots, x_{n}\right) R\right)$ if and only if $f\left(P_{P}\right)=n$.

(v) $f(I)=\min \left\{f\left(P_{P}\right) \mid I \subseteq P \in \operatorname{spec} R\right\}$.

(vi) $f\left(I_{S}\right) \leqslant$ height $I_{S}$. In particular, for $P \in \operatorname{spec} R, f\left(P_{P}\right) \leqslant$ height $P$.

(vii) $f\left(O R_{S}\right)=0$.

Proof. (i), (ii), and (iii) are straightforward. For (iv), if $P \in A\left(\left(x_{1}, \ldots, x_{n}\right) R\right)$, then $P_{P} \in A\left(\left(x_{1}, \ldots, x_{n}\right) R_{P}\right)$ so that $f\left(P_{P}\right)=n$, using (ii). Conversely, if $f\left(P_{P}\right)=n$, then by (ii) we see that $x_{1}, \ldots, x_{n}$ is a maximal $A$-sequence in $P_{P}$. Thus $P_{P} \in$ $A\left(\left(x_{1}, \ldots, x_{n}\right) R_{P}\right)$, so that $P \in A\left(\left(x_{1}, \ldots, x_{n}\right) R\right)$. For (v), if $I \subseteq P \in \operatorname{spec} R$, then by (i) and (iii), $f(I) \leqslant f(P) \leqslant f\left(P_{P}\right)$. Now let $x_{1}, \ldots, x_{n}$ be a maximal $A$-sequence in $I$. By the prime avoidance lemma, we must have some $P \in A\left(\left(x_{1}, \ldots, x_{n}\right) R\right)$ with $I \subseteq P$. By (iv), $f(I)=n=f\left(P_{P}\right)$. Now (vi) follows from Lemma 1.1(ii), and implies (vii).

We now give numerous examples. We will not prove every statement we make about these examples, but will give enough references that the interested reader may pursue matters. Here we define $A(I)$, the definition extending to $A\left(I_{S}\right)$ in the natural way. 
Example 1. $A_{1}(I)=\operatorname{Ass}(R / I)$. The $A_{1}$-sequences are $R$-sequences and the grade function is classical grade.

Recall that for $I$ an ideal, the sequence $\operatorname{Ass}\left(R / I^{n}\right), n=1,2,3, \ldots$, stabilizes for large $n$. We denote that stable set by $A^{*}(I)$ (see [5, Chapter 1]).

ExAmple 2. $A_{2}(I)=A^{*}(I)$. Now in general, neither $\operatorname{Ass}(R / I) \subseteq A^{*}(I)$ nor $A^{*}(I) \subseteq \operatorname{Ass}(R / I)$. That is $A_{1}(I) \nsubseteq A_{1}(I)$ and $A_{2}(I) \nsubseteq A_{1}(I)$. However if $I$ is generated by an $R$-sequence, then $A_{1}(I)=A_{2}(I)$ [1, Exercise 13, p. 103]. From this, one easily sees that the $A_{2}$-sequences and the grade function of $A_{2}$ are identical to those of $A_{1}$.

Ideals $I$ and $J$ are called projectively equivalent, denoted $I \sim J$, if for some positive integers $n$ and $m$ we have $\overline{I^{n}}=\overline{J^{m}}$, where the bar means take the integral closure of the ideal.

ExAmple 3. $A_{3}(I)=\bigcap A^{*}(J)$ over all $J \sim I$. This example and the next are discussed in [ 2 and 6]. The $A_{3}$-sequences are called essential sequences, the grade function is called essential grade.

EXAmple 4. $A_{4}(I)=\left\{P \in \operatorname{spec} R \mid I \subseteq P\right.$, there is a $q \in \operatorname{Ass}\left(R_{P}\right)^{*}$ with $\left(P_{P}\right)^{*}$ minimal over $\left.I\left(R_{P}\right)^{*}+q\right\}$. Here, $\left(R_{P}\right)^{*}$ is the completion of $R_{P}$. In general, $A_{4}(I) \subseteq A_{3}(I)$ (this following easily from [6, Corollary 2]), but $A_{3}(I) \nsubseteq A_{4}(I)$. However, for $I$ an ideal generated by an essential sequence, $A_{3}(I)=A_{4}(I)$. Thus the $A_{4}$-sequences and the grade function for $A_{4}$ are the same as for $A_{3}$.

For $I$ an ideal, and $\bar{I}$ its integral closure, the sequence $\operatorname{Ass}\left(R / \overline{I^{n}}\right), n=1,2,3, \ldots$, stabilizes for large $n$ to a set denoted $\overline{A^{*}}(I)$ (see [5, Chapter 3]). It is known that $\bar{A}^{*}(I) \subseteq A^{*}(I)$, the inclusion of ten being proper.

ExAMPLE 5. $A_{5}(I)=\bar{A}^{*}(I)$. The $A_{5}$-sequences are called asymptotic sequences. The grade function is called asymptotic grade (see [5, Chapter 5]).

EXAMPLE 6. $A_{6}(I)=\left\{P \in \operatorname{spec} R \mid I \subseteq P\right.$, there is a minimal prime $q$ of $\left(R_{P}\right)^{*}$ with $\left(P_{P}\right)^{*}$ minimal over $\left.I\left(R_{P}\right)^{*}+q\right\}$. In general $A_{6}(I) \subseteq A_{5}(I)$ [5, Proposition 3.18] but $A_{5}(I) \nsubseteq A_{6}(I)$. The grade function is again asymptotic grade.

EXAmple 7. $A_{7}(I)=\{P \in \operatorname{spec} R \mid P$ is minimal over $I\}$. The grade function is easily seen to be $f(I)=$ height $I$.

Remarks. (a) By Lemma 1.1(i), $A_{7}(I) \subseteq A_{6}(I)$. Clearly $A_{6}(I) \subseteq A_{4}(I)$, and [6, Proposition 3.3.1] shows $A_{3}(I) \subseteq A_{2}(I)$. Thus if $f_{n}$ is the grade function of example $n$, then $f_{1}=f_{2} \leqslant f_{3}=f_{4} \leqslant f_{5}=f_{6} \leqslant f_{7}$. In general, none of the inequalities are equalities.

(b) Lemma 1.2(vi) shows $f(I) \leqslant$ height $I$. If $f$ is a natura! grade function (i.e. existing on all Noetherian rings) we can ask for which rings will $f(I)=$ height $I$ for all ideals $I$. For $f_{1}=f_{2}$ the answer, of course, is Cohen-Macaulay rings. For $f_{3}=f_{4}$ it is locally unmixed rings (the local $\operatorname{ring}(R, M)$ is unmixed if $\operatorname{depth} q=\operatorname{dim} R$ for all $q \in$ Ass $\left.R^{*}\right)$. For $f_{5}=f_{6}$ the answer is locally quasi-unmixed rings $((R, M)$ is quasi-unmixed if depth $q=\operatorname{dim} R$ for all minimal primes $q$ of $\left.R^{*}\right)$. This is discussed further in $\S 6$.

We saw in the examples that two different grade schemes can have the same grade function. As this will be important to us, we will explore it in the next two lemmas. 
First, however, we will make a remark which will often save us the trouble of worrying about localizations of $R$.

REMARK. If $A$ is a grade scheme on $R$, its domain is the set of all ideals in all localizations of $R$. If $R_{S}$ is a localization of $R$, then $A$ can be restricted to all ideals in all localizations of $R_{S}$, and the result is easily seen to be a grade scheme on $R_{S}$ (called $A$ restricted to $R_{S}$ ). Similar statments hold for proto-grade schemes and grade functions. In many instances we will need to prove results involving arbitrary $R_{S}$, but since we will often have hypotheses which restrict to $R_{S}$, we will just deal with $R$ itself. This is a notational convenience which we will use frequently (usually tacitly). The proof of (i) $\Rightarrow$ (ii) of the next lemma and the proof of Lemma 1.4 are our first applications of this remark.

LEMMA 1.3. Let $A$ and $B$ be two grade schemes on $R$. The following are equivalent.

(i) The grade functions of $A$ and $B$ are equal.

(ii) $x_{1}, \ldots, x_{n}$ is an $A$-sequence if and only if it is a B-sequence.

Proof. (ii) $\Rightarrow$ (i) is obvious. Assume now that (i) holds but (ii) fails, letting $x_{1}, \ldots, x_{n}$ be a minimal counterexample. Without loss, assume this is an $A$-sequence but not a $B$-sequence. Since $x_{1}, \ldots, x_{n-1}$ is an $A$-sequence, by minimality it is also a $B$-sequence. Therefore we must have some $P \in B\left(x_{1}, \ldots, x_{n-1}\right)$ with $x_{n} \in P$. By Lemma 1.2(iv), if $f$ is the common grade function of $A$ and $B$, then we have both $f\left(P_{P}\right)=n-1$ and $P \in A\left(x_{1}, \ldots, x_{n-1}\right)$. This contradicts that $x_{1}, \ldots, x_{n}$ is an $A$ sequence.

REMARK. The reader will note that in the preceding proof we introduced the convention that if $x_{1}, \ldots, x_{n}$ is a sequence in $R, A\left(x_{1}, \ldots, x_{n}\right)$ will be used to denote $A\left(\left(x_{1}, \ldots, x_{n}\right) R\right)$. When doubt exists as to which ring $x_{1}, \ldots, x_{n}$ comes from, we will use the more formal notation.

According to Lemma 1.3, the next definition is unambiguous.

Definition. Let $f$ be a grade function on $R$. Then $x_{1}, \ldots, x_{n}$ is an $f$-sequence if it is an $A$-sequence for any grade scheme $A$ on $R$ whose grade function is $f$.

LEMMA 1.4. Let $A$ be a grade scheme on $R$ whose grade function is $f$. Let $B$ be a proto-grade scheme on $R$. Then the following are equivalent.

(i) $B$ is a grade scheme whose grade function is $f$.

(ii) If $x_{1}, \ldots, x_{n}$ is an $f$-sequence, then $A\left(x_{1}, \ldots, x_{n}\right)=B\left(x_{1}, \ldots, x_{n}\right)$.

Proof. (i) $\Rightarrow$ (ii). Assume (i) holds, and let $x_{1}, \ldots, x_{n}$ be an $f$-sequence. Thus it is both an $A$-sequence and a $B$-sequence. If $P$ is a prime containing $\left(x_{1}, \ldots, x_{n}\right)$, then Lemma 1.2(iv) shows $P \in A\left(x_{1}, \ldots, x_{n}\right)$ if and only if $P \in B\left(x_{1}, \ldots, x_{n}\right)$ (if and only if $\left.f\left(P_{P}\right)=n\right)$.

(ii) $\Rightarrow$ (i). Assuming (ii), we will show that $x_{1}, \ldots, x_{n}$ is an avoiding sequence for $B$ if and only if it is an $A$-sequence. The truth of (i) is then trivial. Since $A(0)=B(0)$ (the case $n=0$ of (ii)), our statement is true for $x_{1}$. Assume inductively that it is true for sequences of length $n-1$. Let $x_{1}, \ldots, x_{n}$ be either an avoiding sequence for $B$ or an $A$-sequence. The same can be said for $x_{1}, \ldots, x_{n-1}$, which by induction is therefore an $A$-sequence. By (ii), $A\left(x_{1}, \ldots, x_{n-1}\right)=B\left(x_{1}, \ldots, x_{n-1}\right)$. By the nature of 
$x_{1}, \ldots, x_{n}$, we see that $x_{n}$ is not in any prime contained in $A\left(x_{1}, \ldots, x_{n-1}\right)=$ $B\left(x_{1}, \ldots, x_{n-1}\right)$, and so $x_{1}, \ldots, x_{n}$ is both an avoiding sequence for $B$ and an $A$-sequence. This completes the proof.

Definition. If $A$ and $B$ are grade schemes on $R$, write $A \subseteq B$ if $A\left(I_{S}\right) \subseteq B\left(I_{S}\right)$ for every ideal $I_{S}$ in every localization $R_{S}$. (Of course it is enough to have $A(I) \subseteq B(I)$ for each ideal $I$ of $R$.)

We next show that any grade function has a minimal grade scheme associated with it.

Definition. By $v(I)$ we will mean the smallest number of elements in a generating set for the ideal $I$. (Note that $f(I) \leqslant$ height $I \leqslant v(I)$.)

LEMMA 1.5. Let $f$ be a grade function on $R$. If $f(I)=v(I)$, then I can be generated by an $f$-sequence (necessarily of length $f(I)$ ).

Proof. This is similar to the proof of [1, Theorem 125].

THEOREM 1.6. Let $f$ be a grade function on $R$. Let $A_{f}^{m}(I)=\{P \in \operatorname{spec} R \mid I \subseteq P$ and either $P$ is minimal over $I$ or $\left.f\left(P_{P}\right)=f\left(I_{P}\right)=v\left(I_{P}\right)\right\}$. Define $A_{f}^{m}\left(I_{S}\right)$ analogously. Then $A_{f}^{m}$ is a grade scheme for $f$, and if $A$ is any grade scheme for $f$, then $A_{f}^{m} \subseteq A$.

Proof. Let $A$ be any grade scheme for $f$. Suppose $P \in A_{f}^{m}(I)$. If $P$ is minimal over $I$, then $P \in A(I)$. Otherwise, $f\left(P_{P}\right)=f\left(I_{P}\right)=v\left(I_{P}\right)$, and by Lemma 1.5 , there is an $f$-sequence $x_{1}, \ldots, x_{n}$ in $R_{P}$ with $I_{P}=\left(x_{1}, \ldots, x_{n}\right) R_{P}$. Since $f\left(P_{P}\right)=f\left(I_{P}\right)=n$, $P_{P} \in A\left(\left(x_{1}, \ldots, x_{n}\right) R_{P}\right)=A\left(I_{P}\right)$, and so $P \in A(I)$. Therefore $A_{f}^{m} \subseteq A$.

We now see that $A_{f}^{m}(I)$ is finite, since it is contained in $A(I)$. We easily see that $A_{f}^{m}$ is a proto-grade scheme, and we will apply Lemma 1.4 to it and $A$. Letting $y_{1}, \ldots, y_{m}$ be an $f$-sequence, we already have $A_{f}^{m}\left(y_{1}, \ldots, y_{m}\right) \subseteq A\left(y_{1}, \ldots, y_{m}\right)$. Conversely, if $P \in A\left(y_{1}, \ldots, y_{m}\right)$, then

$$
f\left(\left(y_{1}, \ldots, y_{m}\right) R_{P}\right)=f\left(P_{P}\right)=m \geqslant v\left(\left(y_{1}, \ldots, y_{m}\right) R_{P}\right) \geqslant f\left(\left(y_{1}, \ldots, y_{m}\right) R_{P}\right) .
$$

Equality holds throughout, showing $P \in A_{f}^{m}\left(y_{1}, \ldots, y_{m}\right)$, and completing our proof.

Lemma 1.7. Let $f$ be a grade function on $R$, and let $I$ and $J$ be ideals. Then $f(I \cap J)=f(I J)=\min \{f(I), f(J)\}$.

Proof. $f(I \cap J)=f(I J)$ by Lemma 1.2(v), since $I \cap J$ and $I J$ have the same radical. Now let $I J \subseteq P \in \operatorname{spec} R$ with $f(I J)=f\left(P_{P}\right)$. Without loss, assume $I \subseteq P$. Then $f(I) \leqslant f\left(P_{P}\right)=f(I J) \leqslant f(I)$, the last step since $I J \subseteq I$. Thus $f(I)=f(I J)$. As $f(I J) \leqslant f(J)$, we are done.

We close this section by noting that if $A(I)=\operatorname{Ass}(R / \bar{I})$, then $A$ is not a grade scheme, in general. [5, pp. 40-41] contains an example of a local ring in which two different maximal avoiding sequences for $A$ have different lengths.

2. Characterizing grade functions. We now turn to a more subtle fact about grade functions. It will be a key property in our characterization of grade functions, for it captures the requirement in a grade scheme, $A(I)$ is finite.

Definition. Let $Q \in \operatorname{spec} R$, and let $U$ be an infinite subset of $\operatorname{spec} R$ such that every $P \in U$ properly contains $Q$. If for any infinite subset $U^{\prime}$ of $U$ we have $\bigcap\left\{P \in U^{\prime}\right\}=Q$, then $(Q, U)$ is called a conforming pair. 
LEMMA 2.1. Let $I$ be an ideal of $R$, and let $U_{0}$ be an infinite subset of $\operatorname{spec} R$ such that $I \subseteq P$ for all $P \in U_{0}$. Then there is a conforming pair $(Q, U)$ with $I \subseteq Q$ and $U \subseteq U_{0}$.

Proof. Expand $I$ to an ideal $Q$ maximal with respect to being properly contained in infinitely many primes of $U_{0}$. It is straightforward to see that $Q$ is prime. If $U=\left\{P \in U_{0} \mid Q \subsetneq P\right\}$, it is straightforward to see that $(Q, U)$ is a conforming pair.

THEOREM 2.2. Let $f$ be a grade function on $R$, and let $(Q, U)$ be a conforming pair in $R$. Suppose $f\left(P_{P}\right) \leqslant n$ for all $P \in U$. Then $f\left(Q_{Q}\right) \leqslant n-1$.

Proof. If not, let $x_{1}, \ldots, x_{n}$ be elements in $R$ whose images in $R_{Q}$ form an $f$-sequence in $Q_{Q}$. Let $A$ be a grade scheme for $f$, and let

$$
W=\bigcup\left\{p \in A\left(x_{1}, \ldots, x_{i-1}\right) \mid x_{i} \in p\right\}
$$

the union over $i=1, \ldots, n$. If $p \in W$, we must have $p \nsubseteq Q$ since otherwise $x_{1}, \ldots, x_{n}$ would not be an $f$-sequence in $Q_{Q}$. As $(Q, U)$ is a conforming pair, if $p \in W$, then at most finitely many $P \in U$ can contain $p$. Since $W$ is finite, by deleting finitely many $P$ from $U$ we may assume that $p \nsubseteq P$ whenever $p \in W$ and $P \in U$. We now see that the images of $x_{1}, \ldots, x_{n}$ form an $f$-sequence in $R_{P}$ for all $P \in U$. Since $f\left(P_{P}\right) \leqslant n$, we have $P_{P} \in A\left(\left(x_{1}, \ldots, x_{n}\right) R_{P}\right)$, so that $P \in A\left(x_{1}, \ldots, x_{n}\right)$ for all $P \in U$. This is impossible, since $U$ is infinite.

REMARK. Using [7, Example 2, pp. 202-205] it is not hard to produce a Noetherian ring $R$ with a conforming pair $(Q, U)$ such that little height $Q=$ little height $P=2$ for all $P \in U$. (Little height $P$ is the length of the shortest saturated chain of primes from $P$ down to a minimal prime.) Thus Theorem 2.2 shows that, in general, little height is not a grade function.

The next corollary combines three results which have appeared in three different places.

COROLlaRY 2.3. Let $f$ be a grade function on $R$ and let $Q \in \operatorname{spec} R$ with $f\left(Q_{Q}\right)=$ height $Q$. Let $W=\{P \in \operatorname{spec} R \mid Q \subset P$ and height $P / Q=1\}$. Then for all but finitely many $P \in W, f\left(P_{P}\right)=$ height $P$. In particular if $R_{Q}$ is Cohen-Macaulay (respectively, unmixed) (respectively, quasi-unmixed) then the same is true of $R_{P}$ for all but finitely many $P \in W$.

Proof. If false, then $W$ contains an infinite subset $U$ with $f\left(P_{P}\right)<$ height $P$ for all $P \in U$. By [4, Theorem 1] height $P=$ height $Q+1$ for all but finitely many $P \in U$, and deleting those finitely many does no harm. Clearly $(Q, U)$ is now a conforming pair with $f\left(P_{P}\right)<$ height $P=$ height $Q+1=f\left(Q_{Q}\right)+1$ for all $P \in U$. By Theorem 2.2, $f\left(Q_{Q}\right)<f\left(Q_{Q}\right)$, a contradiction. The three special cases follow by letting $f$ be, respectively, classical, essential, and asymptotic grade.

We now come to our main result.

THEOREM 2.4. Let $f$ be a nonnegative integer valued function defined on the set $\left\{I_{S} \mid I_{S}\right.$ is a proper ideal in some localization $R_{S}$ of $\left.R\right\}$. Then $f$ is a grade function if and only if it satisfies the following three conditions.

(i) $f\left(I_{S}\right)=\min \left\{f\left(P_{P}\right) \mid I_{S} \subseteq P_{S} \in \operatorname{spec} R_{S}\right\}$. 
(ii) $f\left(P_{P}\right) \leqslant$ height $P$ for all $P \in \operatorname{spec} R$.

(iii) If $(Q, U)$ is a conforming pair in $R$, and if $f\left(P_{P}\right) \leqslant n$ for all $P \in U$, then $f\left(Q_{Q}\right) \leqslant n-1$.

Proof. One direction is by Lemma 1.2(v) and (vi) and Theorem 2.2. Thus assume $f$ satisfies the given conditions. We seek a grade scheme $A_{f}$ whose grade function is $f$. We define $A_{f}\left(I_{S}\right)$ to be $\left\{P_{S} \in \operatorname{spec} R_{S} \mid I_{S} \subseteq P_{S}\right.$ and $\left.f\left(P_{P}\right)=f\left(I_{P}\right)\right\}$. Before proceeding, we note that condition (i) implies that if $I_{S} \subseteq J_{S}$ are ideals, then $f\left(I_{S}\right) \leqslant f\left(J_{S}\right)$ and $f(I) \leqslant f\left(I_{S}\right)$. We will use these inequalities repeatedly.

By condition (i), find a $P \in \operatorname{spec} R$ with $I \subseteq P$ such that $f(I)=f\left(P_{P}\right)$. Then $f(I) \leqslant f\left(I_{P}\right) \leqslant f\left(P_{P}\right)=f(I)$, so that $f\left(I_{P}\right)=f\left(P_{P}\right)$. Thus $P \in A_{f}(I)$ which is therefore nonempty. We also claim $A_{f}(I)$ is finite, since if not, by Lemma 2.1 we could find a conforming pair $(Q, U)$ with $I \subseteq Q$ and $U \subseteq A_{f}(I)$. For all $P \in U$, we would have $f\left(P_{P}\right)=f\left(I_{P}\right)$ By condition (i), $f\left(I_{P}\right) \leqslant f\left(Q_{Q}\right)$. Thus $f\left(P_{P}\right) \leqslant f\left(Q_{Q}\right)$ for all $P \in U$, and condition (iii) gives $f\left(Q_{Q}\right) \leqslant f\left(Q_{Q}\right)-1$, a contradiction. Therefore $A_{f}(I)$ is a finite nonempty set, and by the remark preceding Lemma 1.3 , so is $A_{f}\left(I_{S}\right)$. Also, it is clear from the definition that $P_{S} \in A_{f}\left(I_{S}\right)$ implies $I_{S} \subseteq P_{S}$, and if $P \in \operatorname{spec} R$ with $P \cap S=\varnothing$, then $P \in A_{f}(I)$ if and only if $P_{S} \in A_{f}\left(I_{S}\right)$. Therefore, we have that $A_{f}$ is a proto-grade scheme on $R$.

In order to complete our proof, we will prove the following two statements.

(a) If $x_{1}, \ldots, x_{n}$ is an avoiding sequence for $A_{f}$, and if $P \in A_{f}\left(x_{1}, \ldots, x_{n}\right)$, then $f\left(P_{P}\right)=n$.

(b) If $f(I)=n$, then any maximal avoiding sequence for $A_{f}$ in $I$ has length $n$.

Obviously (b) implies that $A_{f}$ is a grade scheme for $f$, as desired. To prove (b), we will simultaneously treat (a) and (b) by inducting on $n$. For $n=0$, suppose $P \in A_{f}(0)$. Then $f\left(P_{P}\right)=f\left(O R_{P}\right)$. However, applying conditions (i) and (ii) to any minimal prime of $R_{P}$ shows that $f\left(O R_{P}\right)=0$, so (a) holds when $n=0$. As for (b), suppose $f(I)=0$. By condition (i), there is an $I \subseteq P \in \operatorname{spec} R$ with $f\left(P_{P}\right)=0=$ $f\left(O R_{P}\right)$, so that $P \in A_{f}(O)$, clearly showing (b) holds.

Inductively assume both (a) and (b) hold for arbitrary $0 \leqslant m<n$. We will show they hold for $n$. For (a), let $x_{1}, \ldots, x_{n}$ be an avoiding sequence for $A_{f}$, and let $P \in A_{f}\left(x_{1}, \ldots, x_{n}\right)$. If $f\left(P_{P}\right)<n$, then $f(P) \leqslant f\left(P_{P}\right)<n$, and (b) is inductively violated. Thus $f\left(P_{P}\right) \geqslant n$. As $P \in A_{f}\left(x_{1}, \ldots, x_{n}\right), f\left(P_{P}\right)=f\left(\left(x_{1}, \ldots, x_{n}\right) R_{P}\right)$. Let $P_{P}$ be a prime minimal over $\left(x_{1}, \ldots, x_{n}\right) R_{P}$. Then $f\left(P_{P}\right)=f\left(\left(x_{1}, \ldots, x_{n}\right) R_{P}\right) \leqslant f\left(p_{P}\right)$ $\leqslant f\left(p_{p}\right) \leqslant$ height $p \leqslant n$, using condition (ii) and the principal ideal theorem. Thus $f\left(P_{P}\right)=n$ and (a) is inductively proved.

For (b), let $f(I)=n$ and let $x_{1}, \ldots, x_{k}$ be an avoiding sequence for $A_{f}$ in $I$. If $k<n$, then for any $P \in A_{f}\left(x_{1}, \ldots, x_{k}\right)$, statement (a) shows $f\left(P_{P}\right)=k<n=f(I)$, and so condition (i) shows $I \nsubseteq P$. Since $A_{f}\left(x_{1}, \ldots, x_{k}\right)$ is finite, we can find an $x_{k+1} \in I$ with $x_{1}, \ldots, x_{k}, x_{k+1}$ an avoiding sequence for $A_{f}$.

Now let $y_{1}, \ldots, y_{l}$ be a maximal avoiding sequence for $A_{f}$ in $I$. We want $l=n$, and by the preceding paragraph have $l \geqslant n$. By condition (i), there is an $I \subseteq P \in \operatorname{spec} R$ with $f\left(P_{P}\right)=f(I)=n$. Since $l \geqslant n$ we may consider $y_{1}, \ldots, y_{n}$, which is an avoiding sequence for $A_{f}$. To prevent statement (b) from being inductively violated, we must 
have $f\left(y_{1}, \ldots, y_{n}\right) \geqslant n$. Therefore $n \leqslant f\left(\left(y_{1}, \ldots, y_{n}\right) R_{P}\right) \leqslant f\left(P_{P}\right)=n$. This shows that $f\left(P_{P}\right)=f\left(\left(y_{1}, \ldots, y_{n}\right) R_{P}\right)$, so that $P \in A_{f}\left(y_{1}, \ldots, y_{n}\right)$. Since $I \subseteq P$, we now see that $y_{1}, \ldots, y_{n}$ is a maximal avoiding sequence for $A_{f}$ in $I$. Thus $l=n$ as desired. This proves (b), and the theorem.

We examine the grade scheme constructed in Theorem 2.4 a bit more closely, first naming it, and then showing some of its nice properties.

DEFINITION. Let $f$ be a grade function on $R$. For $I_{S}$ an ideal in some localization $R_{S}$ of $R$, let $A_{f}\left(I_{S}\right)=\left\{P_{S} \in \operatorname{spec} R_{S} \mid I_{S} \subseteq P_{S}\right.$ and $\left.f\left(I_{P}\right)=f\left(P_{P}\right)\right\} . A_{f}$ is called the canonical grade scheme of $f$.

REMARK. It is easily seen that $A_{f}(I)=\{P \in \operatorname{spec} R \mid$ there is an ideal $J$ with $\left.J \subseteq I \subseteq P \in A_{f}^{m}(J)\right\}$.

LEMMA 2.5. Let $A_{f}$ be the canonical grade scheme of the grade function $f$ on $R$. Let $I \subseteq J \subseteq P \subseteq Q$ and $K$ be ideals of $R$ with $P$ and $Q$ prime.

(a) If $P \in A_{f}(I)$, then $P \in A_{f}(J)$.

(b) If $f(I)=f\left(P_{P}\right)$, then $Q \in A_{f}(I)$ if and only if $Q \in A_{f}(J)$, in which case $f(I)=f\left(Q_{Q}\right)$.

(c) If $f(I)=f\left(P_{P}\right)$, then $P$ is maximal in $A_{f}(I)$ if and only if $P$ is maximal in $A_{f}(J)$.

(d) The hypothesis $f(I)=f\left(P_{P}\right)$ in (b) and (c) hold if I is generated by an $f$-sequence and $P \in A_{f}(I)$.

(e) $P \in A_{f}(I K)$ if and only if either (i) $K \nsubseteq P$ and $P \in A_{f}(I)$ or (ii) $K \subseteq P$ and $P \in A_{f}(I) \cap A_{f}(K)$.

Proof. (a) $f\left(P_{P}\right) \geqslant f\left(J_{P}\right) \geqslant f\left(I_{P}\right)=f\left(P_{P}\right)$, the equality since $P \in A_{f}(I)$. Thus equality holds throughout, and $P \in A_{f}(J)$.

(b) If $Q \in A_{f}(I)$, then $Q \in A_{f}(J)$ by (a). If $Q \in A_{f}(J)$, then $f\left(Q_{Q}\right)=f\left(J_{Q}\right) \leqslant$ $f\left(J_{P}\right) \leqslant f\left(P_{P}\right)=f(I) \leqslant f\left(I_{Q}\right) \leqslant f\left(Q_{Q}\right)$, which shows $Q \in A_{f}(I)$ and also $f(I)=$ $f\left(Q_{Q}\right)$.

(c) This follows easily from (a) and (b) since $f(I)=f\left(P_{P}\right)$ gives $P \in A_{f}(I)$.

(d) This is easy.

(e) If (i) holds, then $(I K)_{P}=I_{P}$, and since $P \in A_{f}(I)$ we have $P_{P} \in A_{f}\left(I_{P}\right)=$ $A_{f}\left((I K)_{P}\right)$. Thus $P \in A_{f}(I K)$. If (ii) holds, then $f\left(I_{P}\right)=f\left(P_{P}\right)=f\left(K_{P}\right)$. Lemma 1.6 now shows $P \in A_{f}(I K)$. Conversely, suppose $P \in A_{f}(I K)$. If $K \nsubseteq P$, then $P_{P} \in A_{f}\left((I K)_{P}\right)=A_{f}\left(I_{P}\right)$, so $P \in A_{f}(I)$. If $K \subseteq P$, then since $I K \subseteq K \subseteq P$, part (a) shows $P \in A_{f}(K)$. Similarly $P \in A_{f}(I)$.

We will now identify the canonical grade scheme $A_{f}$ when $f$ is asymptotic grade. We will use the following definition.

Definition. For $P$ prime in a Noetherian ring $R$,

$$
z(P)=\min \left\{\operatorname{depth} q \mid q \text { is a minimal prime in }\left(R_{P}\right)^{*}\right\},
$$

with $\left(R_{P}\right)^{*}$ the completion of $R_{P}$.

THEOREM 2.6. Let $f$ be asymptotic grade on $R$. Let $I \subseteq P \in \operatorname{spec} R$. Then $P \in A_{f}(I)$ if and only if for each minimal prime $q$ of $\left(R_{P}\right)^{*}$, height $\left(I\left(R_{P}\right)^{*}+q / q\right) \geqslant z(P)$. In particular, $A_{f} \subseteq A_{6}$. 
Proof. By [5, Proposition 6.10] we have both $f\left(P_{P}\right)=z(P)$ and $f\left(I_{P}\right)=$ $\min \left\{\right.$ height $\left(I\left(R_{P}\right)^{*}+q / q\right) \mid q$ is a minimal prime in $\left.\left(R_{P}\right)^{*}\right\}$. Noting that we always have $f\left(I_{P}\right) \leqslant f\left(P_{P}\right)$ and that equality holds exactly when $P \in A_{f}(I)$, the first part of the result is clear. Now suppose $P \in A_{f}(I)$, and let $q$ be a minimal prime in $\left(R_{P}\right)^{*}$ such that depth $q=z(P)$. By the preceding, height $\left(I\left(R_{P}\right)^{*}+q / q\right) \geqslant \operatorname{depth} q$. As the reverse inequality is obvious, we have equality. Thus $\left(P_{P}\right)^{*}$ is minimal over $I\left(R_{P}\right)^{*}+q$. By definition, this gives $P \in A_{6}$. Therefore $A_{f} \subseteq A_{6}$.

In constructing Example $A_{6}$, we used all minimal primes of $\left(R_{P}\right)^{*}$. We now show that a single minimal prime of depth $z(P)$ would suffice.

TheOREM 2.7. For each prime $P$ of $R$, let $q(P)$ be a minimal prime in $\left(R_{P}\right)^{*}$, with depth $q(P)=z(P)$. Define $A\left(I_{S}\right)=\left\{P_{S} \in \operatorname{spec} R_{S} \mid I_{S} \subseteq P_{S}\right.$ and $\left(P_{P}\right)^{*}$ is minimal over $\left.I\left(R_{P}\right)^{*}+q(P)\right\}$. Then $A$ is a grade scheme for asymptotic grade on $R$. Also $A$ contains the canonical grade scheme for asymptotic grade.

Proof. Let $f$ be asymptotic grade on $R$. If $P_{S} \in A_{f}\left(I_{S}\right)$, then $P_{S} \in A\left(I_{S}\right)$ by the argument used in proving Theorem 2.6, with $q=q(P)$. Now $A$ is easily seen to be a proto-grade scheme $\left(A\left(I_{S}\right)\right.$ being finite since it is contained in $\left.A_{6}\left(I_{S}\right)\right)$. Let $x_{1}, \ldots, x_{n}$ be an asymptotic sequence. We have $A_{f}\left(x_{1}, \ldots, x_{n}\right) \subseteq A\left(x_{1}, \ldots, x_{n}\right) \subseteq A_{6}\left(x_{1}, \ldots, x_{n}\right)$ $=A_{f}\left(x_{1}, \ldots, x_{n}\right)$, the equality by Lemma 1.4. As equality holds throughout, Lemma 1.4 shows that $A$ is a grade scheme for $f$.

EXAMPLE. We wish to show that with $f$ being asymptotic grade, in general $A_{6} \nsubseteq A_{f}$. In fact we will show $A \nsubseteq A_{f}$ with $A$ as constructed in Theorem 2.7. This is very easy. Let $(R, M)$ be a complete local ring with exactly two minimal primes $q_{1}$ and $q_{2}$, both of depth 1 . Let $q(M)=q_{1}$. Since $M$ is minimal over $q_{2}+q(M)$, $M \in A\left(q_{2}\right)$. However, height $\left(q_{2}+q_{2} / q_{2}\right)=0<1=z(M)$, and so Theorem 2.6 shows $M \notin A_{f}\left(q_{2}\right)$.

REMARK. The preceding two theorems and example concern asymptotic grade and minimal primes of $\left(R_{P}\right)^{*}$. Analogous results hold for essential grade and prime divisors of $\left(R_{P}\right)^{*}$, using [6, Corollary 5.4].

EXAMPLE. We now let $f$ be classical grade and give examples showing that in general $A_{f}$ is not contained in $A_{1}$ or $A_{2}$, and $A_{1}$ and $A_{2}$ are not contained in $A_{f}$.

Let $(R, M)$ be a 2-dimensional local domain which is not Cohen-Macaulay, and let $P$ be a height 1 prime. Since $f\left(M_{M}\right)=1=f\left(P_{M}\right), M \in A_{f}(P)$. Clearly $M \notin$ $A_{1}(P)$. Thus $A_{f} \nsubseteq A_{1}$.

Let $R$ be a Cohen-Macaulay domain and let $P$ be a prime such that for some $n, P^{n}$ is not $P$-primary. Then for some $P \subsetneq Q \in \operatorname{spec} R, Q \in A_{1}\left(P^{n}\right)$. However $f\left(Q_{Q}\right)=$ height $Q>$ height $P=f\left(P_{Q}^{n}\right)$, so $Q \notin A_{f}\left(P^{N}\right)$. Thus $A_{1} \nsubseteq A_{f}$.

Let $(R, M)$ be a 2-dimensional local domain which is not Cohen-Macaulay, but which is unmixed (i.e. every $q \in$ Ass $R^{*}$ has depth $q=2$ ). For any ideal $I \neq 0$, $f\left(I_{M}\right)=1=f\left(M_{M}\right)$ so that $M \in A_{f}(I)$ for all $I \neq 0$. However since $R$ is unmixed, [5, Proposition 10.11] shows for some $I \neq 0, M \notin A_{2}(I)$. Thus $A_{f} \nsubseteq A_{2}$.

Let $(R, M)$ be a 2-dimensional normal domain, and let $P$ be a nonprincipal height 1 prime. By [5, Corollary 4.7], $M \in A_{2}(P)$. However, being 2-dimensional and 
normal, $R$ is Cohen-Macaulay. Therefore, $f(M)=2>1=f\left(P_{M}\right)$, and so $M \notin$ $A_{f}(P)$. Thus $A_{2} \nsubseteq A_{f}$.

We now show that the canonical grade scheme of a grade function can often be enlarged in a natural way.

Definition. Let $f$ be a grade function on $R$. If $q$ is an ideal of $R$, we will say that $q$ is $f$-modest if whenever $q \subseteq P \in \operatorname{spec} R$, we have $f\left(P_{P}\right) \leqslant \operatorname{height}(P / q)$.

REMARKS. (i) If $q$ is an ideal in the nilradical of $R$, then $q$ is easily seen to be $f$-modest for any grade function $f$.

(ii) If $f$ is asymptotic grade and $q$ is a minimal prime of $R$, then $q$ is $f$-modest using [5, Proposition 6.10].

(iii) If $f$ is essential grade and $q \in$ Ass $R$, then $q$ is $f$-modest using [6, Corollary 5.4].

(iv) If $f$ is classical grade and $q \in$ Ass $R$, then $q$ is $f$-modest by (iii) and the fact that classical grade does not exceed essential grade.

(v) Let $q$ be $f$-modest and let $Q$ be a prime minimal over $q$. Then $f\left(Q_{Q}\right) \leqslant$ height $Q / q=0$. So $f\left(Q_{Q}\right)=0$ and $Q \in A_{f}(0)$. (This does not characterize $f$-modesty. In fact $Q$ may not be $f$-modest.)

(vi) If every prime minimal over $Q$ is $f$-modest, then $q$ is $f$-modest (but not conversely), as is easily seen.

LeMMA 2.8. Let $f$ be a grade function on $R$, and let $q$ be an $f$-modest ideal of $R$. Let $x_{1}, \ldots, x_{n}$ be an $f$-sequence in $R$ with $\left(x_{1}, \ldots, x_{n}\right)+q \neq R$. Then

(a) $f\left(\left(x_{1}, \ldots, x_{n}\right)+q\right)=n$,

(b) $A_{f}\left(\left(x_{1}, \ldots, x_{n}\right)+q\right)=\left\{P \in A_{f}\left(x_{1}, \ldots, x_{n}\right) \mid q \subseteq P\right\}$.

Proof. (a) Let $P$ be minimal over $\left(x_{1}, \ldots, x_{n}\right)+q$. Then $n \leqslant f\left(\left(x_{1}, \ldots, x_{n}\right)+q\right)$ $\leqslant f\left(P_{P}\right) \leqslant$ height $P / q \leqslant n$, using $f$-modesty and the principal ideal theorem.

(b) One containment is by Lemma $2.5(\mathrm{a})$. Conversely, if $P \in A_{f}\left(\left(x_{1}, \ldots, x_{n}\right)+q\right)$, then $f\left(P_{P}\right)=f\left(\left(x_{1}, \ldots, x_{n}\right)_{P}+q_{P}\right)=n=f\left(\left(x_{1}, \ldots, x_{n}\right)_{P}\right)$ showing $P \in$ $A_{f}\left(x_{1}, \ldots, x_{n}\right)$. Here we used part (a) applied in $R_{P}$ to get the second equality.

THEOREM 2.9. Let $f$ be a grade function on $R$. Let $V$ be a finite set of $f$-modest ideals of $R$. Define $A_{f V}\left(I_{S}\right)=A_{f}\left(I_{S}\right) \cup\left(\cup A_{f}\left(I_{S}+q_{S}\right)\right)$ over all $q \in V$ with $I_{S}+q_{S} \neq R_{S}$. Then $A_{f V}$ is a grade scheme for $f$.

Proof. It is easily seen that $A_{f V}$ is a proto-grade scheme. We will use Lemma 1.4. Let $x_{1}, \ldots, x_{n}$ be an $f$-sequence. We want $A_{f}\left(x_{1}, \ldots, x_{n}\right)=A_{f v}\left(x_{1}, \ldots, x_{n}\right)$. One inclusion is by definition of $A_{f V}$, and the other is by Lemma 2.8(b).

Let $f$ be asymptotic grade. We previously saw $A_{f} \subseteq A_{6} \subseteq A_{5}$. If $V$ is the set of minimal primes in $R$, we now give an example in which $A_{f V} \nsubseteq A_{5}$.

EXAmple. Let $R$ be $K[[X, Y, Z, W]]$ modulo $(X) \cap(Y, Z)$. Let $x, y, z, w$ be the images of $X, Y, Z, W$, and let $M$ be the maximal ideal of $R$. Let $V=\{(x),(y, z)\}$. Now let $I=(y, w)$. We claim that $M \in A_{f V}(I)$. It will suffice to show that $M \in A_{f}(I+(x))$. Note that

$$
\operatorname{height}(I+(x) /(x))=\operatorname{height}(I+(x)+(y, z) /(y, z))=2=z(M) .
$$


By Theorem 2.6, $M \in A_{f}(I+(x))$, so that $M \in A_{f V}(I)$. We now claim that $M \notin A_{5}(I)=\overline{A^{*}}(I)$. If it were, then by [5, Proposition 3.18] we would have either $M /(x) \in \bar{A}^{*}(I+(x) /(x))$ or $M /(y, z) \in \bar{A}^{*}(I+(y, z) /(y, z))$. However, since $R /(x)$ and $R /(y, z)$, being complete local domains, satisfy the altitude formula, [5, Proposition 4.1] would give either $3=$ height $M /(x)=l(I+(x) /(x)) \leqslant 2$ (since the analytic spread of an ideal does not exceed its minimal number of generators) or $2=$ height $M /(y, z)=l(I+(y, z) /(y, z))=1$. Thus $M \notin A_{5}(I)$, and so $A_{f V} \nsubseteq$ $A_{5}$.

EXAMPLE. Let $B$ be an arbitrary grade scheme for $f$ and let $V$ be a finite set of $f$-modest ideals. In analogy to $A_{f V}$, we can try to define

$$
B_{V}\left(I_{S}\right)=B\left(I_{S}\right) \cup\left(\cup B\left(I_{S}+q_{S}\right)\right)
$$

over all $q \in V$ with $I_{S}+q_{S} \neq R_{S}$. However, in general the result will not be a grade scheme for $f$. In fact, it may not be a grade scheme at all, as we now show, using an example in which $B$ differs from $A_{f}$ by very little. Let $(R, M)$ be $K[X, Y, Z]$ localized at $(X, Y, Z)$. Let $q=(X)$ and $p=(X, Y)$. Theorem 2.4 makes it clear that in order to define a grade function on $R$, it suffices to specify its values on $P_{P}$ for all $P \in \operatorname{spec} R$, in such a way that conditions (ii) and (iii) of Theorem 2.4 are satisfied. Thus we let $f\left(P_{P}\right)=$ height $P$, with the following exceptions: $f\left(q_{q}\right)=0, f(M)=2$, and $f\left(P_{P}\right)=1$ whenever $P$ is a height 2 prime containing $q$. We see that $q$ is $f$-modest, and will let $V=\{0, q\}$. We now let $B\left(I_{S}\right)=A_{f}\left(I_{S}\right)$ in all cases except $B(p)$ which we take to be $A_{f}(p) \cup\{M\}=\{p, M\}$. (Note that by condition (i) of Theorem 2.4, $f(p)=f\left(p_{p}\right)=1$, and since $f(M)=2, M \notin A_{f}(p)$.) Since $f(p)=1$ $<$ height $p, p$ is not generated by an $f$-sequence. Since $B$ and $A_{f}$ agree everywhere but at $p$, Lemma 1.4 shows that $B$ is a grade scheme for $f$. We now claim that $B_{V}$, defined as above, is not a grade scheme. (It clearly is a proto-grade scheme.) We first note that $B_{V}(0)=B(0) \cup B(q)=A_{f}(0) \cup A_{f}(q)=\{0, q\}$. Thus $Y$ and $Z$ are both avoiding sequences for $B_{V}$. We will show that $Y$ is maximal in $M$, but $Z$ is not, showing that $B_{V}$ is not a grade scheme. Since $B_{V}(Y)=B(Y) \cup B((Y)+q)=B(Y)$ $\cup B(p)$, and since $M \in B(p), Y$ is a maximal avoiding sequence for $B_{V}$. On the other hand $B_{V}(Z)=B(Z) \cup B((Z)+q)=A_{f}(Z) \cup A_{f}((Z, X))$, which does not contain $M$, since $f(M)=2$ while $f(Z)=1=f((Z, X))$.

3. Factor rings. We mentioned that to define a grade function $f$, it suffices to specify $f\left(P_{P}\right)$ for all $P \in \operatorname{spec} R$ in such a way that conditions (ii) and (iii) of Theorem 2.4 hold. We use this (on $R / q$ ) in our next theorem.

THEOREM 3.1. Let $f$ be a grade function on $R$ and let $q$ be an $f$-modest ideal. For $P / q \in \operatorname{spec}(R / q)$, let $h\left(P_{P} / q_{P}\right)=f\left(P_{P}\right)$. Then $h$ defines a grade function on $R / q$. If $J / q$ is an ideal of $R / q$, then $h(J / q)=f(J)$. Also $P \in A_{f}(J)$ if and only if $P / q \in A_{h}(J / q)$.

Proof. Since $q$ is $f$-modest, $h\left(P_{P} / q_{P}\right)=f\left(P_{P}\right) \leqslant \operatorname{height}(P / q)$. Thus condition (ii) of Theorem 2.4 holds for $h$. As for condition (iii), let $(Q / q, U)$ be a conforming pair in $R / q$ and suppose $h\left(P_{P} / q_{P}\right) \leqslant n$ for all $P / q \in U$. We want $h\left(Q_{Q} / q_{Q}\right) \leqslant n-1$. Obviously $(Q,\{P \mid P / q \in U\})$ is a conforming pair in $R$, and for these $P, f\left(P_{P}\right)=$ $h\left(P_{P} / q_{P}\right) \leqslant n$. Therefore $f\left(Q_{Q}\right) \leqslant n-1$, and so $h\left(Q_{Q} / q_{Q}\right) \leqslant n-1$ as desired. 
Now $h$ is seen to give a grade function on $R / q$ defined via condition (i) of Theorem 2.4. If $J / q$ is an ideal in $R / q$, then

$$
\begin{aligned}
h(J / q) & =\min \left\{h\left(P_{P} / q_{p}\right) \mid J / q \subseteq P / q \in \operatorname{spec}(R / q)\right\} \\
& =\min \left\{f\left(P_{P}\right) \mid J \subseteq P \in \operatorname{spec} R\right\}=f(J) .
\end{aligned}
$$

Finally, $P \in A_{f}(J)$ if and only if $f\left(P_{P}\right)=f\left(J_{P}\right)$ if and only if $h\left(P_{P} / q_{P}\right)=h\left(J_{P} / q_{P}\right)$ (by applying the preceding sentence in $R_{P}$ and $\left.R_{P} / q_{P}\right)$ if and only if $P / q \in A_{h}(J / q)$.

REMARK. Let $B$ be a grade scheme for $f$, and for $J / q$ in $R / q$ let $B_{q}(J / q)=$ $\{P / q \mid P \in B(J)\}$. If $B=A_{f}$, Theorem 3.1 shows that $B_{q}=A_{h}$. However in general, $B_{q}$ will not be a grade scheme at all, as the example at the end of $\$ 2$ works here as well.

Corollary 3.2. Let $f, q$, and $h$ be as in Theorem 3.2. Let $x_{1}, \ldots, x_{n}$ be an $f$-sequence with $\left(x_{1}, \ldots, x_{n}\right)+q \neq R$. Then

(a) $x_{1}+q, \ldots, x_{n}+q$ is an $h$-sequence.

(b) $A_{h}\left(x_{1}+q, \ldots, x_{n}+q\right)=\left\{P / q \mid q \subseteq P \in A_{f}\left(x_{1}, \ldots, x_{n}\right)\right\}$.

Proof. We induct on $n$ simultaneously for (a) and (b). When $n=0$, (a) is trivial. For (b), suppose $q \subseteq P$. Then $P \in A_{f}(0)$ if and only if $f\left(P_{P}\right)=0$ if and only if $h\left(P_{P} / q_{P}\right)=0$ if and only if $P / q \in A_{h}(0)$.

Now suppose (a) and (b) both hold for $n-1$. We will show they both hold for $n$. Suppose this fails for (a). Then $x_{1}+q, \ldots, x_{n}+q$ is not an $h$-sequence, but by induction, $x_{1}+q, \ldots, x_{n-1}+q$ is. Thus for some $P / q \in A_{h}\left(x_{1}+q, \ldots, x_{n-1}+q\right)$, we must have $x_{n}+q \in P / q$. By (b), inductively, $P \in A_{f}\left(x_{1}, \ldots, x_{n-1}\right)$. As $x_{n} \in P$, we have contradicted that $x_{1}, \ldots, x_{n}$ is an $f$-sequence.

As for (b), since by (a) we already have that $x_{1}+q, \ldots, x_{n}+q$ is an $h$-sequence, if $P / q \in A_{h}\left(x_{1}+q, \ldots, x_{n}+q\right)$, then $h\left(P_{P} / q_{P}\right)=n$. Thus $f\left(P_{P}\right)=n$. As $x_{1}, \ldots, x_{n}$ is an $f$-sequence in $P_{P}$, we see that $P \in A_{f}\left(x_{1}, \ldots, x_{n}\right)$. The reverse is similar.

We already noted that if $q$ is in the nilradical of $R$, then $q$ is $f$-modest for any grade function $f$. We will show that for such $q$, Theorem 3.1 gives a bijection between all grade functions on $R$ and all grade functions on $R / q$.

LEMMA 3.3. Let $q$ be an ideal in the nilradical of $R$ and let $f$ be a grade function on $R$. For any ideal $I$ of $R, f(I)=f(I+q)$ and $A_{f}(I)=A_{f}(I+q)$.

Proof. For some $I \subseteq P \in \operatorname{spec} R, f(I)=f\left(P_{P}\right)$. As $q \subseteq P, f(I) \leqslant f(I+q) \leqslant$ $f\left(P_{P}\right)=f(I)$, and equality holds throughout. Thus $f(I)=f(I+q)$. Also, $P \in A_{f}(I)$ if and only if $f\left(P_{P}\right)=f\left(I_{P}\right)$ if and only if $f\left(P_{P}\right)=f\left(I_{P}+q_{P}\right)$ (since $q_{P}$ is in the nilradical of $\left.R_{P}\right)$ if and only if $P \in A_{f}(I+q)$.

THEOREM 3.4. Let $q$ be an ideal contained in the nilradical of $R$.

(a) The map $f \rightarrow h$ (as in Theorem 3.1) is a bijection between the set of grade functions on $R$ and the set of grade functions on $R / q$.

(b) If $f \rightarrow h$ is as above, then $P \in A_{f}(I)$ if and only if $P / q \in A_{h}(I+q / q)$.

(c) If $f \rightarrow h$ is as above, $x_{1}, \ldots, x_{n}$ is an $f$-sequence if and only if $x_{1}+q, \ldots, x_{n}+q$ is an h-sequence.

(d) The bijection in (a) takes asymptotic grade on $R$ to asymptotic grade on $R / q$. 
Proof. (a) We will construct an inverse map for the map $f \rightarrow h$. Suppose that $k$ is a grade function on $R / q$, and define $l$ as follows. For $P$ any prime in $R$, we define $l\left(P_{P}\right)$ to equal $k\left(P_{P} / q_{P}\right)$. We claim that $l$ is a grade function on $R$. This is proved using Theorem 2.4 and is similar to the proof of Theorem 3.1. Now start with $k$ and define $l$ as above. Then let $f=l$ and define $h$ as in Theorem 3.1. We have $h(J / q)=f(J)=l(J)=k(J+q / q)=k(J / q)$ (since $q \subseteq J)$. That is, $h=k$. Conversely, start with $f$ and $h$ as in Theorem 3.1, let $k=h$ and define $l$ as above. We see that $l(I)=k(I+q / q)=h(I+q / q)=f(I+q)=f(I)$ by Lemma 3.3. That is, $l=f$ and (a) is proved.

(b) Immediate from Lemma 3.3 and the final sentence of Theorem 3.1.

(c) One direction is by Corollary 3.2(a). Thus suppose that $x_{1}+q, \ldots, x_{n}+q$ is an $h$-sequence, but that $x_{1}, \ldots, x_{n}$ is not an $f$-sequence. Then for some $i=1,2, \ldots, n$ there is a $P \in A_{f}\left(x_{1}, \ldots, x_{i-1}\right)$ with $x_{i} \in P$. By (b), $P / q \in A_{h}\left(x_{1}+q, \ldots, x_{i-1}+q\right)$. As $x_{i}+q \in P / q$, we have a contradiction.

(d) In view of (c), we need only show that $x_{1}, \ldots, x_{n}$ is an asymptotic sequence in $R$ if and only if $x_{1}+q, \ldots, x_{n}+q$ is an asymptotic sequence in $R / q$. For this, note that $P \in \bar{A}^{*}(I)$ if and only if there is a minimal prime $Q \subseteq P$ with $P / Q \in$ $\bar{A}^{*}(I+Q / Q)\left[5\right.$, Proposition 3.18], from which it easily follows that $P \in \bar{A}^{*}(I)$ if and only if $P / q \in \bar{A}^{*}(I+q / q)$. Using this, our statement about asymptotic sequences is straightforward.

We will now show that grade functions on certain collections of factor rings of $R$ induce a grade function on $R$. Then we will combine this with Theorem 3.1.

THEOREM 3.5. Let $V$ be a finite set of ideals of $R$, and suppose for any $P \in \operatorname{spec} R$ there is a $q \in V$ with $q \subseteq P$. For each $q \in V$, let $f_{q}$ be a grade function on $R / q$. Define $f^{\prime}$ in the following way. For $I_{S}$ an ideal in some localization $R_{S}$ of $R, f^{\prime}\left(I_{S}\right)=$ $\min \left\{f_{q}\left(I_{S}+q_{S} / q_{S}\right) \mid q \in V, I_{S}+q_{S} \neq R_{S}\right\}$. Then $f^{\prime}$ is a grade function on $R$.

Proof. Note that the hypothesis on $V$ assures that for any $I_{S}$, there is a $q \in V$ with $I_{S}+q_{S} \neq R_{S}$, so that $f^{\prime}\left(I_{S}\right)$ is defined. We will show that $f^{\prime}$ satisfies Theorem 2.4. For condition (i), we need only to consider an ideal $I$ in $R$ itself. Say $I \subseteq P \in \operatorname{spec} R$. Then

$$
\begin{aligned}
f^{\prime}\left(P_{P}\right) & =\min \left\{f_{q}\left(P_{P} / q_{P}\right) \mid q \in V, q \subseteq P\right\} \\
& \geqslant \min \left\{f_{q}(I+q / q) \mid q \in V, q \subseteq P\right\} \geqslant f^{\prime}(I) .
\end{aligned}
$$

We must now show $f^{\prime}\left(P_{P}\right)=f^{\prime}(I)$ for some $P$. For some $q \in V$, we have $f^{\prime}(I)=$ $f_{q}(I+q / q)$, and for some $I+q / q \subseteq P / q \in \operatorname{spec} R / q, f_{q}(I+q / q)=f_{q}\left(P_{P} / q_{P}\right)$. As $f_{q}\left(P_{P} / q_{P}\right) \geqslant f^{\prime}\left(P_{P}\right) \geqslant f^{\prime}(I)$, combining the above shows this is the $P$ we seek.

Condition (ii) is easy, since $f^{\prime}\left(P_{P}\right)=\min \left\{f_{q}\left(P_{P} / q_{P}\right) \mid q \in V, q \subseteq P\right\} \leqslant$ $\min \{$ height $P / q \mid q \in V, q \subseteq P\} \leqslant$ height $P$.

As for condition (iii), let $(Q, U)$ be a conforming pair in $R$, with $f^{\prime}\left(P_{P}\right) \leqslant n$ for all $P \in U$. Thus for each $P \in U$, there is a $q \in V, q \subseteq P$, with $f_{q}\left(P_{P} / q_{P}\right)=f^{\prime}\left(P_{P}\right) \leqslant n$. As $U$ is infinite while $V$ is finite, we find that for some fixed $q \in V$ there are infinitely many $P \in U$ for which $q \subseteq P$ and $f_{q}\left(P_{P} / q_{P}\right) \leqslant n$. Deleting the other $P$ 's 
from $U$ does no harm, as we still have a conforming pair. We now have $q \subseteq P$ for all $P \in U$, and so $q \subseteq Q$. It is clear that $(Q / q,\{P / q \mid P \in U\})$ is a conforming pair in $R / q$, and since $f_{q}\left(P_{P} / q_{P}\right) \leqslant n$ for all such $P / q$, we have $f_{q}\left(Q_{Q} / q_{Q}\right) \leqslant n-1$. Thus $f^{\prime}\left(Q_{Q}\right) \leqslant f_{q}\left(Q_{Q} / q_{Q}\right) \leqslant n-1$, as desired.

COROllaRY 3.6. Let $R, V, f_{q}$, and $f^{\prime}$ be as in Theorem 3.5.

(a) If $P \in A_{f^{\prime}}(I)$, then for some $q \in V$ with $q \subseteq P, P / q \in A_{f_{q}}(I+q / q)$.

(b) If for all $q \in V$ with $q \subseteq P$ we have $P / q \in A_{f_{q}}(I+q / q)$, then $P \in A_{f^{\prime}}(I)$.

(c) If $I$ is generated by an $f^{\prime}$-sequence, then $P \in A_{f^{\prime}}(I)$ if and only if for some $q \in V$ with $q \subseteq P, P / q \in A_{f_{q}}(I+q / q)$.

(d) If $x_{1}, \ldots, x_{n}$ is an $f^{\prime}$-sequence, and if $q \in V$ with $\left(x_{1}, \ldots, x_{n}\right)+q \neq R$, then $x_{1}+q, \ldots, x_{n}+q$ is an $f_{q}$-sequence.

(e) If $R$ is a local ring, then $x_{1}, \ldots, x_{n}$ is an $f^{\prime}$-sequence if and only if $x_{1}+q, \ldots, x_{n}$ $+q$ is an $f_{q}$-sequence for each $q \in V$.

Proof. For (a), let $P \in A_{f^{\prime}}(I)$, so that $f^{\prime}\left(P_{P}\right)=f^{\prime}\left(I_{P}\right)$. By definition of $f^{\prime}$, $f^{\prime}\left(I_{P}\right)=f^{\prime}\left(P_{P}\right)=f_{q}\left(P_{P} / q_{P}\right) \geqslant f_{q}\left(I_{P}+q_{P} / q_{P}\right) \geqslant f^{\prime}\left(I_{P}\right)$ for some $q \in V$ with $q \subseteq$ $P$. Therefore equality holds throughout, and (a) is true. For (b), it is easily seen that we may assume $R$ is local at $P$. Now the hypothesis shows $f_{q}(P / q)=f_{q}(I+q / q)$ for all $q \in V$. The definition of $f^{\prime}$ now shows that $f^{\prime}(P)=f^{\prime}(I)$ so that $P \in A_{f^{\prime}}(I)$. To prove (c) and (d), we argue similarly to the proof of Corollary 3.2. As for (e), one direction is by (d), while the other is any easy induction using (a).

REMARK. The converse of Corollary 3.6(a) fails even when $R$ is local. Let $(R, M)$ be 1-dimensional with $V=\{q, Q\}$ being exactly the minimal primes of $R$. Let $f_{q}$ and $f_{Q}$ be classical grade. Let $I=q$. Now $f_{Q}(M / Q)=1=f_{Q}(I+Q / Q)$ so that $M / Q$ $\in A_{Q}(I+Q / Q)$. However $f^{\prime}(M)=\min \left\{f_{q}(M / q), f_{Q}(M / Q)\right\}=1$ while $f^{\prime}(I)=$ $\min \left\{f_{q}(q / q), f_{Q}(q+Q / Q)\right\}=0$. Thus $M \notin A_{f^{\prime}}(I)$. The next corollary corrects this situation, at the cost of possibly going to a larger grade scheme. That is, if the $B_{q}$ in Corollary 3.7 all equal the canonical grade scheme $A_{q}$ of $f_{q}$, then the $B$ produced wil have $A_{f^{\prime}} \subseteq B$.

COROLlaRY 3.7. Let $R, V, f_{q}$ and $f^{\prime}$ be as in Theorem 3.5, and suppose that for each $q \in V, B_{q}$ is a grade scheme for $f_{q}$. Define $B\left(I_{S}\right)=\left\{P_{S} \in \operatorname{spec} R_{S} \mid I_{S} \subseteq P_{S}\right.$, and for some $q \in V$ with $\left.q \subseteq P, P_{S} / q_{S} \in B_{q}\left(I_{S}+q_{S} / q_{S}\right)\right\}$. Then $B$ is a grade scheme for $f^{\prime}$.

Proof. It is straightforward to verify that $B$ is a proto-grade scheme. We will invoke Lemma 1.4 applied to $A_{f^{\prime}}$ and $B$. Thus let $x_{1}, \ldots, x_{n}$ be an $f^{\prime}$-sequence. We must show that $A_{f^{\prime}}\left(x_{1}, \ldots, x_{n}\right)=B\left(x_{1}, \ldots, x_{n}\right)$. Suppose $P \in A_{f^{\prime}}\left(x_{1}, \ldots, x_{n}\right)$. By Corollary 3.6(a), for some $q \in V$ with $q \subseteq P, P / q \in A_{f_{q}}\left(x_{1}+q, \ldots, x_{n}+q\right)$. However by (d) of that corollary, $x_{1}+q, \ldots, x_{n}+q$ is an $f_{q}$-sequence, so that $P / q \in$ $B_{q}\left(x_{1}+q, \ldots, x_{n}+q\right)$ by Lemma 1.4. By definition $P \in B\left(x_{1}, \ldots, x_{n}\right)$, so that $A_{f^{\prime}}\left(x_{1}, \ldots, x_{n}\right) \subseteq B\left(x_{1}, \ldots, x_{n}\right)$. The reverse containment is proved similarly, using Corollary 3.6(c).

THEOREM 3.8. Let $V$ be a finite set of ideals of $R$ such that every prime of $R$ contains some $q \in V$. 
(a) Let $f$ be a grade function on $R$ such that every $q \in V$ is $f$-modest. For each $q \in V$ define $h_{q}$ on $R / q$ as in Theorem 3.1. Now letting $f_{q}=h_{q}$, define $f^{\prime}$ as in Theorem 3.5. Then $f^{\prime}=f$.

(b) For each $q \in V$ let $f_{q}$ be a grade function on $R / q$, and define $f^{\prime}$ as in Theorem 3.5. Then every $q \in V$ is $f^{\prime}$-modest. Now letting $f=f^{\prime}$, define $h_{q}$ on $R / q$ as in Theorem 3.1. Then $f_{q} \geqslant h_{q}$.

Proof. (a) For any ideal $I$ of $R, f^{\prime}(I)=\min \left\{f_{q}(I+q / q) \mid q \in V, I+q \neq R\right\}$. Since $f_{q}=h_{q}$, the characterization of $h_{q}$ in Theorem 3.1 gives

$$
f^{\prime}(I)=\min \{f(I+q) \mid q \in V, I+q \neq R\} .
$$

We will show that $f(I)$ equals the same minimum. Clearly it is equal to or less than that minimum. Now there is a prime $P$ containing $I$ with $f(I)=f\left(P_{P}\right)$. Since $P$ contains some $q \in V, f(I) \leqslant f(I+q) \leqslant f\left(P_{P}\right)=f(I)$, and equality holds throughout.

(b) If $q \in V$ and $q \subseteq P \in \operatorname{spec} R$, then $f^{\prime}\left(P_{P}\right) \leqslant f_{q}\left(P_{P} / q_{P}\right) \leqslant$ height $P / q$ since $f_{q}$ is a grade function. Thus $q$ is $f^{\prime}$-modest. Now for an ideal $J / q$ in $R / q, h_{q}(J / q)=$ $f(J)=f^{\prime}(J)=\min \left\{f_{Q}(J+Q / Q) \mid Q \in V, J+Q \neq R\right\}$. Letting $Q=q$ shows $h_{q}(J / q) \leqslant f_{q}(J / q)$.

4. Extensions. Recall that rings $R \subseteq T$ satisfy lying over if for every $P \in \operatorname{spec} R$, there is a $p \in \operatorname{spec} T$ with $p \cap R=P$.

Lemma 4.1. Let the Noetherian rings $R \subseteq T$ satisfy lying over. Then for every $P \in \operatorname{spec} R$, there is a $p \in \operatorname{spec} T$ with $p \cap R=P$ and height $p \leqslant$ height $P$.

Proof. Let $S=R-P$, and let $a_{1}, \ldots, a_{n}$ be a system of parameters in $R_{P}=R_{S}$ (so that height $P=n$ ). By lying over, we have $\left(a_{1}, \ldots, a_{n}\right) T_{S} \neq T_{s}$. If $p_{S} \in \operatorname{spec} T_{S}$ with $p_{S}$ minimal over $\left(a_{1}, \ldots, a_{n}\right) T_{S}$, clearly $p \cap R=P$ and height $p \leqslant n$ by the principal ideal theorem.

THEOREM 4.2. Let $R \subseteq T$ be Noetherian rings which satisfy lying over. Let $g$ be a grade function on $T$. For $I_{S}$ any ideal in any localization $R_{S}$ of $R$, let $f\left(I_{S}\right)$ be defined to be $g\left(I T_{S}\right)$. Then

(a) $f$ is a grade function on $R$.

(b) If $P \in A_{f}(I)$, then there is a $p \in A_{g}(I T)$ with $p \cap R=P$.

(c) If $I$ is generated by an $f$-sequence, then the converse of (b) also holds.

(d) Elements $x_{1}, \ldots, x_{n}$ in $R$ are an $f$-sequence if and only if they are a g-sequence.

(e) Let $B$ be any grade scheme for $g$. For $I_{S}$ any ideal in any localization $R_{S}$ of $R$, let $C\left(I_{S}\right)=\left\{P_{S} \in \operatorname{spec} R_{S} \mid\right.$ there is a $p_{s} \in B\left(I T_{S}\right)$ with $\left.p_{S} \cap R_{S}=P_{S}\right\}$. Then $C$ is a grade scheme for $f$.

(f) If $T$ is a faithfully flat extension of $R$ and if $g$ is any of classical, essential, or asymptotic grade, $f$ is the same.

(g) If $T$ is an integral extension of $R$ such that every minimal prime of $T$ contracts to a minimal prime of $R$, and if $g$ is asymptotic grade, so is $f$. 
(h) If $T$ is a finite module extension of $R$ such that every $p \in$ Ass $T$ has $p \cap R \in$ Ass $R$, and if $g$ is essential grade, so is $f$.

Proof. (a) First note that if $I$ is an ideal of $R$ and $S$ is a multiplicatively closed subset of $R$ disjoint from $I$, our hypothesis easily shows that $I T \cap S=\varnothing$. Thus $f\left(I_{S}\right)=g\left(I T_{S}\right)$ is defined. Furthermore, it is easily seen that for $P \in \operatorname{spec} R$, $f\left(P_{P}\right)=\min \left\{g\left(p_{p}\right) \mid p \in \operatorname{spec} T, p \cap R=P\right\}$. Using this, it is not hard to see that $f$ satisfies condition (i) of Theorem 2.4. It also satisfies condition (ii) of that theorem, using Lemma 4.1. For condition (iii), let $(Q, U)$ be a conforming pair in $R$, with $f\left(P_{P}\right) \leqslant n$ for all $P \in U$. We want $f\left(Q_{Q}\right) \leqslant n-1$. For each $P \in U$, the preceding shows there is a $p \in \operatorname{spec} T$ with $p \cap T=P$ and $g\left(p_{p}\right)=f\left(P_{P}\right) \leqslant n$. For each $P \in U$, consider one such $p \in \operatorname{spec} T$, and let $W$ denote the set of those $p$. Clearly $Q T \subseteq p$ for all $p \in W$, and so by Lemma 2.1 there is a conforming pair $\left(q, W^{\prime}\right)$ of $T$ with $Q T \subseteq q$ and $W^{\prime} \subseteq W$. As $g\left(p_{p}\right) \leqslant n$ for all $p \in W^{\prime}$, we have that $g\left(q_{q}\right) \leqslant n-$ 1. By the preceding, in order to show that $f\left(Q_{Q}\right) \leqslant n-1$, it will suffice to show that $q \cap R=Q$. Clearly $Q \subseteq q \cap R$. Conversely, $q \cap R \subseteq \cap\left\{p \cap R \mid p \in W^{\prime}\right\}$. By construction, $\left\{p \cap R \mid p \in W^{\prime}\right\}$ is an infinite subset of $U$, and so $q \cap R \subseteq Q$ as desired.

(b) Let $P \in A_{f}(I)$. Then $f\left(P_{P}\right)=f\left(I_{P}\right)$. As noted in the proof of (a), there is a $p \in \operatorname{spec} T, p \cap R=P$, with $g\left(p_{p}\right)=f\left(P_{P}\right)$. If $S=R-P$, then $f\left(I_{P}\right)=g\left(I T_{S}\right) \leqslant$ $g\left(I T_{p}\right) \leqslant g\left(p_{p}\right)=f\left(P_{P}\right)=f\left(I_{P}\right)$. Equality holds throughout, showing $p \in A_{g}(I T)$.

(c) and (d) We prove these simultaneously inducting on $n$. For $n=0$, (d) is trivial. For (c), say $p \in A_{g}(0)$ and $p \cap R=P$. Then $g\left(p_{p}\right)=0$, and so $f\left(P_{P}\right)=0$, showing $P \in A_{f}(0)$. Now suppose (c) and (d) both hold for $n-1$. Then the case $n$ of (d) follows easily from (b) and the cases $n-1$ of (c) and (d). As for the case $n$ of (c), say $p \in A_{g}\left(x_{1}, \ldots, x_{n}\right)$, with $p \cap R=P$ and $x_{1}, \ldots, x_{n}$ an $f$-sequence. By the case $n$ of (d), these are also a $g$-sequence. Thus $g\left(p_{p}\right)=n$, so that $f\left(P_{P}\right) \leqslant n$. As $x_{1}, \ldots, x_{n}$ are in $P_{P}, f\left(P_{P}\right)=n$ and $P \in A_{f}\left(x_{1}, \ldots, x_{n}\right)$.

(e) Clearly $C$ is a proto-grade scheme. Let $x_{1}, \ldots, x_{n}$ be an $f$-sequence, so that by part (d), it is also a $g$-sequence. $P \in C\left(x_{1}, \ldots, x_{n}\right)$ if and only if there is a $p \in B\left(x_{1}, \ldots, x_{n}\right)$ with $p \cap R=P$ if and only if there is a $p \in A_{g}\left(x_{1}, \ldots, x_{n}\right)$ with $p \cap R=P$ (using Lemma 1.4 applied to $B$ and $A_{g}$ ) if and only if $P \in A_{f}\left(x_{1}, \ldots, x_{n}\right)$ (by part (c)). Thus Lemma 1.4 applied to $C$ and $A_{f}$ shows that $C$ is a grade scheme for $f$.

(f) With $T$ a faithfully flat extension of $R$ and $I$ an ideal of $R$, it is well known that classical grade $I=$ classical grade $I T$. Also essential grade $I=$ essential $I T$ by [6, Proposition 5.6.1]. That the same is true of asymptotic grade is shown in [10, Corollary 5.2].

(g) This follows easily from [5, Proposition 3.22].

(h) This is given by [6, Proposition 5.6.2].

Let $R \subseteq T$ be a faithfully flat extension of Noetherian rings. Let $p \in \operatorname{spec} T$ and let $P=p \cap R$. It is easily seen that $T_{p}$ is an extension of $R_{P}$, and furthermore it follows from [7, 19.2(2)] that $R_{P} \subseteq T_{p}$ satisfies lying over. In the proof of the next corollary we shall start with $h$ being one of classical, essential, or asymptotic grade on $T$ (hence also on $T_{p}$ ) and we will apply Theorem 4.2 to $R_{P} \subseteq T_{p}$ and $g=h$ on $T_{p}$. 
A key step in that proof will be to show that the resulting grade function $f$ on $R_{P}$ also equals $h$. We do that now as a lemma.

Lemma 4.3. Let $R \subseteq T$ be a faithfully flat extension of Noetherian rings. Let $p \in \operatorname{spec} T$ and let $P=p \cap R$. Let $h$ be any one of classical, essential or asymptotic grade. Let $f$ be the grade function on $R_{P}$ obtained by applying Theorem 4.2 to $R_{P} \subseteq T_{p}$ starting with $g=h$ on $T_{p}$. Then $f=h$ on $R_{P}$.

Proof. Let $S=R-P$ and let $q \in \operatorname{spec} T$ with $q \subseteq p$ and $q$ minimal over $P T$. Let $T_{1}=T_{S}, T_{2}=T_{p}$ and $T_{3}=T_{q}$. Note that $T_{i+1}$ is a localization of $T_{i}, i=1,2$. Let $f_{i}$ be the grade function on $R_{P}$ obtained by applying Theorem 4.2 to $R_{P} \subseteq T_{i}$, starting with $g=h$ on $T_{i}, i=1,2,3$. Now $R_{P} \subseteq T_{1}$ is a faithfully flat extension, and so Theorem 4.2(f) shows that $f_{1}=h$. Also [7, 19.2(2)] shows that $R_{P} \subseteq T_{3}$ is a faithfully flat extension, and so we also have $f_{3}=h$. To prove our lemma, we will show $f_{1} \leqslant f_{2} \leqslant f_{3}$, giving $f_{2}=h$ as desired. Let $x_{1}, \ldots, x_{n}$ be an $f_{1}$-sequence in $R_{P}$. Theorem 4.2(d) applied to $R_{P} \subseteq T_{1}$ shows that $x_{1}, \ldots, x_{n}$ is an $h$-sequence in $T_{1}$. As $T_{2}$ is a localization of $T_{1}, x_{1}, \ldots, x_{n}$ is also an $h$-sequence in $T_{2}$. Theorem 4.2(d) applied to $R_{P} \subseteq T_{2}$ now shows that $x_{1}, \ldots, x_{n}$ is an $f_{2}$-sequence in $R_{P}$. Thus any $f_{1}$-sequence is also an $f_{2}$-sequence, and so clearly $f_{1} \leqslant f_{2}$. The proof that $f_{2} \leqslant f_{3}$ is identical.

COROllaRY 4.4. Let $R \subseteq T$ be a faithfully flat extension of Noetherian rings. Let $h$ be any of classical, essential, or asymptotic grade. Then if $I$ is an ideal of $R$ and $I \subseteq P \in \operatorname{spec} R$, the following are equivalent.

(a) $P \in A_{h}(I)$.

(b) There is a $p \in A_{h}(I T)$ with $p \cap R=P$.

(c) If $p \in \operatorname{spec} T$ is minimal over $P T$, then $p \in A_{h}(I T)$.

Proof. (a) $\Rightarrow$ (c). Let the prime $p$ be minimal over $P T$. By $[7,19.2(2)], T_{p}$ is a faithfully flat extension of $R_{P}$. If in Theorem 4.2 applied to $R_{P} \subseteq T_{p}$, we start with $g=h$ on $T_{p}$, then the resulting $f$ on $R_{P}$ is also $h$ as is shown by part (f) of that theorem. Now if $P \in A_{h}(I)$, then $P_{P} \in A_{h}\left(I_{P}\right)=A_{f}\left(I_{P}\right)$, and so Theorem 4.2(a) shows that some prime in $A_{g}\left(I T_{P}\right)=A_{h}\left(I T_{p}\right)$ lies over $P_{P}$. However $p_{p}$ is the only prime of $T_{p}$ lying over $P_{P}$, and so we must have $p \in A_{h}(I T)$. Thus (a) $\Rightarrow$ (c).

(c) $\Rightarrow$ (b) is trivial from $[6,18.11]$.

(b) $\Rightarrow$ (a) Let $p \in A_{h}(I T)$ with $p \cap R=P$. We can apply Theorem 4.2 to $R_{P} \subseteq T_{p}$, starting with $g=h$ on $T_{p}$. If $f$ is the resulting grade function on $R_{P}$, then $f=h$ by Lemma 4.3. We now have $h\left(I_{P}\right) \leqslant h\left(P_{P}\right)=f\left(P_{P}\right)=g\left(P_{P} T_{P}\right)=h\left(P T_{p}\right) \leqslant$ $h\left(p_{p}\right)=h\left(I T_{p}\right)$ (since $\left.p \in A_{h}(I T)\right)=g\left(I_{P} T_{p}\right)=f\left(I_{p}\right)=h\left(I_{p}\right)$. As equality must hold throughout, $P \in A_{h}(I)$.

Remark. Corollary 4.4 remains true if $A_{h}$ is replaced by $A_{1}, A_{4}$, or $A_{5}$. For $A_{1}$, use [7, 18.11]. For $A_{4}$ use [6, Proposition 3.7]. For $A_{5}$ use [9, Theorems 8.5 and 8.8]. (It may well also hold for $A_{2}, A_{3}$, and $A_{6}$, but we have not checked.)

EXAMPLE. Corollary 4.4 shows that the converse of Theorem 4.2(b) holds if $R \subseteq T$ is faithfully flat and $g$ is one of classical, essential, or asymptotic grade. We now show that the converse fails for arbitrary $g$, even if $R \subseteq T$ is faithfully flat. Let 
( $R, M$ ) be a 2-dimensional local domain with $P$ a height 1 prime. Let $T=R[X], X$ an indeterminate. Define $g$ by specifying $g\left(p_{p}\right)=$ height $p$ for all $p \in \operatorname{spec} T$ except, for $p=M T$, let $g\left(p_{p}\right)=1$ and, for $p=(P, X)$, let $g\left(p_{p}\right)=0$. With $f$ as in Theorem 4.2, it is easily seen that $M T \in A_{g}(P T)$ but $M \notin A_{f}(P)$.

We now look at integral extensions and will show that $A_{4}$ and $A_{5}$ are better behaved than the canonical grade schemes for essential and asymptotic grade. We first note that if $I$ is an ideal in $R$ and if the Noetherian ring $T$ is an integral extension of $R$ such that every minimal prime of $T$ contracts to a minimal prime of $R$, then $P \in A_{5}(I)$ if and only if there is a $p \in A_{5}(I T)$ with $p \cap R=P_{S}$ [5, Proposition 3.22]. Similarly, if $T$ is a finite module extension of $R$ and every prime in Ass $T$ contracts to a prime in Ass $R$, then $P \in A_{4}(I)$ if and only if there is a $p \in A_{4}(I T)$ with $p \cap R=T$ [6, Proposition 3.9]. We will now give an example showing that neither of these holds for $A_{f}$, with $f$ asymptotic grade and essential grade, respectively. (Of course one direction does hold, by Theorem 4.2(b).)

EXAMPLE. Let $T$ be $K[X, Y]$ localized at the complement of $(X, Y) \cup(X, Y+1)$. Thus $T$ has exactly two maximal ideals $N_{1}$ and $N_{2}$. Now $R=K+\left(N_{1} \cap N_{2}\right)$ is a local ring, its maximal ideal being $M=N_{1} \cap N_{2}$. Also $T$ is a finite $R$-module. Furthermore on $R$ and $T$, essential and asymptotic grades are equal, both being just height. We will call this $f$. Let $p$ be a height 1 prime of $T$ not in $N_{1}$. Let $I=p \cap N_{1}$, an ideal of both $T$ and $R$. Since $I T_{N_{1}}=N_{1}, N_{1} \in A_{f}(I T)$. However $M=N_{1} \cap R \notin$ $A_{f}(I)$, since $f(M)=2$ while $f(I)=1$.

THEOREM 4.5. Let $f$ be a grade function on $R$, and let $X$ be an indeterminate. If $P$ is a prime of $R[X]$ with $P \cap R=p$, then define $g\left(P_{P}\right)$ as follows. If $P=p R[X]$, let $g\left(P_{P}\right)=f\left(p_{p}\right)$. If $P \neq p R[X]$, let $g\left(P_{P}\right)=f\left(p_{p}\right)+1$. Then

(a) $g$ determines a grade function on $R[X]$ (via condition (i) of Theorem 2.4).

(b) If $I$ is an ideal in $R$, then $P \in A_{g}(I R[X])$ if and only if $P=(P \cap R) R[X]$ and $P \cap R \in A_{f}(I)$.

(c) If $a_{1}, \ldots, a_{n}$ are elements in $R$, they are an $f$-sequence if and only if they are a $g$-sequence. Also if $I$ is an ideal of $R$, then $f(I)=g(I R[X])$.

(d) If $f$ is either classical or asymptotic grade on $R$, then $g$ is the same on $R[X]$.

Proof. (a) We must show that $g$ satisfies conditions (ii) and (iii) of Theorem 2.4. Condition (ii) is easy, since it holds for $f$ and since with $P$ and $p$ as in the statement of this theorem, height $P=$ height $p$ if $P=p R[X]$ while height $P=$ height $p+1$ if $P \neq p R[X]$. For condition (iii), we let $(Q, U)$ be a conforming pair in $R[X]$, and assume $g\left(P_{P}\right) \leqslant n$ for all $P \in U$. We need $g\left(Q_{Q}\right) \leqslant n-1$, and will proceed in cases. Case 1 . There is some $p \in \operatorname{spec} R$ such that infinitely many $P \in U$ satisfy $P \cap R=p$. Then we easily see that $Q=p R[X]$. Choose $P \in U$ with $P \cap R=p$ and $P \neq p R[X]$. Then $n \geqslant g\left(P_{P}\right)=f\left(p_{p}\right)+1$. As $g\left(Q_{Q}\right)=f\left(p_{p}\right) \leqslant n-1$, we are done. Case 2. If Case 1 fails, then it does no harm to delete from $U$ the finitely many $P$ satisfying $P \cap R=Q \cap R$. We now partition $U$ into $U_{1}=\{P \in U \mid P=(P \cap R) R[X]\}$ and $U_{2}=\{P \in U \mid P \neq(P \cap R) R[X]\}$. One of $U_{1}$ or $U_{2}$ must be infinite. Subcase 2a. $U_{1}$ is infinite. Recalling our deletions from $U$, we easily see that $(Q \cap R,\{P \cap R \mid P \in$ $\left.U_{1}\right\}$ ) is a conforming pair in $R$. Furthermore, if $q=Q \cap R$, we see that $Q=q R[X]$, since any polynomial in $Q$ has all of its coefficients in $(P \cap R) R[X]$ for all $P \in U_{1}$. 
With $P \in U_{1}, n \geqslant g\left(P_{P}\right)=f\left(p_{p}\right)$ (with $p=P \cap R$ ), and our conforming pair shows that $f\left(q_{q}\right) \leqslant n-1$. Thus $g\left(Q_{Q}\right)=f\left(q_{q}\right) \leqslant n-1$. Subcase 2 b. $U_{2}$ is infinite. Now we see that $\left(q,\left\{P \cap R \mid P \in U_{2}\right\}\right)$ is a conforming pair. If $P \in U_{2}$ and $p=P \cap R$, then $g\left(P_{P}\right) \leqslant n$ gives $f\left(p_{p}\right)=g\left(P_{P}\right)-1 \leqslant n-1$, and so we get $f\left(q_{q}\right) \leqslant n-2$. Since $g\left(Q_{Q}\right) \leqslant f\left(q_{q}\right)+1$, we again have $g\left(Q_{Q}\right) \leqslant n-1$. This completes the proof of part (a).

(b) Let $P \in A_{g}(I R[X])$ and let $p=P \cap R$. We have $g\left(P_{P}\right)=g\left(I R[X]_{P}\right)=$ $\min \left\{g\left(Q_{Q}\right) \mid I R[X]_{P} \subseteq Q_{P} \in \operatorname{spec} R[X]_{P}\right\}$. Taking the particular case $Q=p R[X]$, we get $g\left(P_{P}\right) \leqslant g\left(Q_{Q}\right)=f\left(p_{p}\right)$. Since $p=P \cap R$, the definition of $g$ now shows that $P=p R[X]$. It remains to show that $p \in A_{f}(I)$. We have $g\left(I R[X]_{P}\right)=g\left(P_{P}\right)$ $=f\left(p_{p}\right)$; call this number $n$. Letting $f\left(I_{p}\right)=m$, we want $n=m$, and clearly have $n \geqslant m$ since $I_{p} \subseteq p_{p}$. Now there is some $I_{p} \subseteq q_{p} \in \operatorname{spec} R_{p}$ with $f\left(q_{q}\right)=f\left(I_{p}\right)=m$. With $Q=q R[X]$, we have $I R[X]_{P} \subseteq Q_{P}$, and so $n=g\left(I R[X]_{P}\right) \leqslant g\left(Q_{Q}\right)=f\left(q_{q}\right)$ $=m$. Thus $n=m$. This proves half of (b). The other half is similar to the last part of the above.

(c) This is easy using (b).

(d) We will assume that $f$ is asymptotic grade, the case of classical grade being similar. By [5, Proposition 3.21], if $I$ is an ideal of $R$ then $\bar{A}^{*}(I R[X])=\{p R[X] \mid p$ $\left.\in \bar{A}^{*}(I)\right\}$. Thus if $a_{1}, \ldots, a_{n}$ are elements of $R$, they are an asymptotic sequence in $R$ if and only if they are an asymptotic sequence in $R[X]$. We want $g=f$ on $R[X]$. It is enough to show $f\left(P_{P}\right)=g\left(P_{P}\right)$ for any $P \in \operatorname{spec} R[X]$. If $p=P \cap R$, clearly we may assume that $R$ is local at $p$. Let $a_{1}, \ldots, a_{n}$ be a maximal asymptotic sequence in $p$. Thus $f(p)=n$ and so $g\left(P_{P}\right)=n$ if $P=p R[X]$, while $g\left(P_{P}\right)=n+1$ if $P \neq$ $p R[X]$. We must show the same dichotomy applies to $f\left(P_{P}\right)$. If $P=p R[X]$, we have already noted that since $p \in \bar{A}^{*}\left(a_{1}, \ldots, a_{n}\right), P \in \bar{A}^{*}\left(\left(a_{1}, \ldots, a_{n}\right) R[X]\right)$ and so $f\left(P_{P}\right)$ $=n$. Now suppose $P \neq p R[X]$. Then there is a monic polynomial $h(X)$ with $P=(p R[X], h(X))$. Our previous statements show that $a_{1}, \ldots, a_{n}, h(X)$ is an asymptotic sequence in $P$, so that $f\left(P_{P}\right) \geqslant n+1$. Now letting $P^{\prime}=p R[X]$, since $P^{\prime} \in \bar{A}^{*}\left(\left(a_{1}, \ldots, a_{n}\right) R[X]\right), f\left(P_{P}^{\prime}\right)=n$. By [5, Proposition 5.17] applied to $R[X]_{P}$ and $P_{P}^{\prime}$, and since $P_{P}$ is the only prime larger than $P_{P}^{\prime}$, we see that $f\left(P_{P}\right)=f\left(P_{P}^{\prime}\right)+1$ $=n+1$.

COROllaRY 4.6. Let $X$ be an indeterminate over $R$, and let $f$ be either classical or asymptotic grade. Let $I$ be an ideal of $R$. Then $A_{f}(I R[X])=\left\{p R[X] \mid p \in A_{f}(I)\right\}$.

Proof. This follows immediately from parts (b) and (d) of Theorem 4.5.

REMARK. Theorem $4.5(\mathrm{~d})$ and Corollary 4.6 probably hold for essential grade as well. To mimic the above proofs, we would need for $I$ an ideal of $R$ and $A=A_{3}$ (alternatively $\left.A=A_{4}\right)$ that $A(I R[X])=\{p R[X] \mid p \in A(I)\}$. This is probably true for one of (both of?) $A_{3}$ or $A_{4}$, but we have not checked.

QuEstion. Let $R \subseteq T$ be a faithfully flat extension of Noetherian rings and let $f$ be a grade function on $R$. For $P \in \operatorname{spec} T$ with $p=P \cap R$, define $g\left(P_{P}\right)=f\left(p_{p}\right)+$ height $(P / p T)$. Does $g$ determine a grade function on $T$ ?

REMARK. Let $R \subseteq T$ be an integral extension of Noetherian rings, and let $f$ be a grade function on $R$. For $P \in \operatorname{spec} T$ with $P \cap R=p$, let $g\left(P_{P}\right)=f\left(p_{p}\right)$. It is easily seen that $g$ satisfies condition (iii) of Theorem 2.4. However it may not satisfy 
condition (ii) (since possibly height $P<$ height $p$ ), and therefore $g$ may not determine a grade function on $T$. If every minimal prime of $T$ contracts to a minimal prime of $R$, and if $f$ is asymptotic grade on $R$, then $g$ will be a grade function [5, Proposition 5.14], but need not be asymptotic grade. If

$$
h\left(P_{P}\right)=\min \left\{f\left(p_{p}\right), \text { height } p\right\},
$$

then $h$ always defines a grade function on $T$.

5. Another grade function. We start with an easy consequence of Theorems 3.5 and 4.2, mentioning two known special cases. We then introduce a third special case which we feel deserves attention, but about which we know very little.

Proposition 5.1. Let $(R, M)$ be a local ring with completion $R^{*}$. Let $V$ be a finite set of ideals of $R^{*}$ such that every prime ideal of $R^{*}$ contains some $q \in V$. For each $q \in V$, let $f_{q}$ be a grade function on $R^{*} / q$. For $I_{S}$ any ideal in any localization $R_{S}$ of $R$, define $f\left(I_{S}\right)$ to be $\min \left\{f_{q}\left(I R_{S}^{*}+q_{S} / q_{S}\right) \mid q \in V, I_{S}+q_{S} \neq R_{S}^{*}\right\}$. Then

(a) $f$ is a grade function on $R$.

(b) If $V=\left\{q \in \operatorname{spec} R^{*} \mid q\right.$ is minimal $\}$ and if each $f_{q}$ is the height function, then $f$ is asymptotic grade.

(c) If $V=$ Ass $R^{*}$ and if each $f_{q}$ is the height function, then $f$ is essential grade.

Proof. To prove (a), combine Theorems 3.5 and 4.2. For (b) and (c), use [5, Proposition 5.10 and 6, Corollary 5.4] respectively.

Let $(R, M)$ be a local ring with completion $R^{*}$. Let $V=$ Ass $R^{*}$, and for every $q \in V$, let $f_{q}$ be the classical grade function on $R^{*} / q$. Define $f$ as in Proposition 5.1(a). Thus $f$ is a grade function on $R$. This section reveals our ignorance, and is devoted to questions concerning $f$.

QUESTION. Is there a natural grade function, defined on any Noetherian ring $R$, which coincides with $f$ whenever $R$ is local?

A possible candidate for a grade function answering the previous question is given by the next question.

Question. For $I_{S}$ any ideal in any localization $R_{S}$ of a Noetherian ring $R$, define $g\left(I_{S}\right)$ to be $\min \left\{\operatorname{classical} \operatorname{grade}\left(P_{P}\right)^{*} / q \mid I_{S} \subseteq P_{S} \in \operatorname{spec} R_{S}\right.$, and $\left.q \in \operatorname{Ass}\left(R_{P}\right)^{*}\right\}$. Is $g$ a grade function? If $R$ is local, will $g$ equal the above $f$ ?

The preceding question is motivated by a comparison of [5, Proposition 5.6] to [5, Proposition 6.10], as well as by comparing [6, Proposition 4.3] to [6, Corollary 5.4].

Recall now that $A_{1}$ and $A_{2}$ were defined in our earlier examples.

QuESTION. For $I_{S}$ any ideal in any localization $R_{S}$ of $R$, let $B\left(I_{S}\right)=\left\{P_{S} \in\right.$ $\operatorname{spec} R_{S} \mid I_{S} \subseteq P_{S}$, and there is a $q \in \operatorname{Ass}\left(R_{P}\right)^{*}$ with

$$
\left.\left(P_{P}\right)^{*} / q \in A_{1}\left(\left(I\left(R_{P}\right)^{*}+q\right) / q\right)\right\} \text {. }
$$

If $R$ is local, is $B$ a grade scheme for our $f$ ? Is it a grade scheme at all? What if $R$ is arbitary? What if in the definition of $B$ we replace $A_{1}$ by $A_{2}$ ?

REMARK. We do not know if the $B$ of the preceding question is a grade scheme. However combining Corollary 3.7 and Theorem 4.2(e) shows that for a local ring $(R, M)$ with completion $R^{*}$, we can construct a grade scheme $C$ for the above $f$ in 
the following way. Let $C(I)=\left\{P \in \operatorname{spec} R \mid\right.$ there is a $p \in \operatorname{spec} R^{*}$ with $p \cap R=P$, and a $q \in$ Ass $R^{*}$ with $q \subseteq p$, such that $\left.p / q \in A_{1}\left(I R^{*}+q / q\right)\right\}$ and let $C\left(I_{S}\right)$ be defined appropriately. (Of course $A_{2}$ would do as well as $A_{1}$.)

\section{Modulation.}

Definition. Let $f$ be a grade function on a local ring $(R, M)$. If for every ideal $I$ and element $a \in M, f(I, a) \leqslant f(I)+1$, then we say $f$ is modulated.

Definition. The grade function $f$ on $R$ is locally modulated if $f$ restricted to $R_{P}$ is modulated for every $P \in \operatorname{spec} R$.

RemarKs. (a) It is easy to produce examples in which $f$ restricted to $R_{M}$ is modulated for all maximal ideals $M$, but for which $f$ is not locally modulated.

(b) Let $f$ be any of classical, essential, or asymptotic grade. Then $f$ is locally modulated, [1, Theorem 127; 6, Proposition 5.8 and 5, Proposition 5.16].

THEOREM 6.1. Let $f$ be a grade function on $R$. The following are equivalent.

(a) $f$ is locally modulated.

(b) If $P \subset Q$ are primes of $R$ and height $(Q / P)=1$, then $f\left(Q_{Q}\right) \leqslant f\left(P_{P}\right)+1$.

Proof. (a) $\Rightarrow$ (b) Suppose (a) holds, and $P \subset Q$ are as in (b). Pick $a \in Q_{Q}-P_{Q}$. As $Q_{Q}$ is the only prime of $R_{Q}$ containing $\left(P_{Q}, a\right)$, we have that $f\left(P_{Q}, a\right)=f\left(Q_{Q}\right)$. Now (a) implies $f\left(Q_{Q}\right) \leqslant f\left(P_{Q}\right)+1$. As the only primes containing $P_{Q}$ are $P_{Q}$ and $Q_{Q}$, we have $f\left(P_{Q}\right)=\min \left\{f\left(P_{P}\right), f\left(Q_{Q}\right)\right\}$. Whichever is the minimum, we see that (b) holds.

(b) $\Rightarrow$ (a) Suppose (b) holds. To prove (a), we may assume that $R$ is local. Let $I$ be an ideal and $a$ be a nonunit. For some $I \subseteq p \in \operatorname{spec} R, f(I)=f\left(p_{p}\right)$. If $a \in p$, then $f(I, a) \leqslant f\left(p_{p}\right)=f(I)$ and we are done. If $a \notin p$, let $q$ be a prime minimal over $(p, a)$. Then height $q / p=1$, and by (b), $f\left(q_{q}\right) \leqslant f\left(p_{p}\right)+1$. As $(I, a) \subseteq q, f(I, a)$ $\leqslant f\left(q_{q}\right) \leqslant f\left(p_{p}\right)+1=f(I)+1$, and we are done.

Corollary 6.2. Let $f$ be a locally modulated grade function on $R$, and let $P_{0} \subset P_{1} \subset \cdots \subset P_{n}$ be a saturated chain of primes in $R$ with $P_{0}$ a minimal prime. Then there is a saturated chain of primes $P_{0}=p_{0} \subset \cdots \subset p_{n-1} \subset P_{n}$ with $f\left(p_{i_{p_{i}}}\right)=i$ $=$ height $p_{i}$ for $i=1, \ldots, n-1$.

Proof. Since $P_{0}$ is minimal, $f\left(P_{0_{P_{0}}}\right)=0$. If $n=1$, we are done. If $n>1$, then let $W=\left\{p \in \operatorname{spec} R \mid P_{0} \subset p \subset P_{2}\right.$ is saturated $\}$. By [8, Proposition 2.2], $W$ is infinite. As $f$ is locally modulated, $f\left(p_{p}\right) \leqslant 1$ for all $p \in W$. Let $W_{0}=\left\{p \in W \mid f\left(p_{p}\right)=0\right\}$. If $W_{0}$ is infinite, then $\left(P_{0}, W_{0}\right)$ is a conforming pair, forcing $f\left(P_{0_{P_{0}}}\right)<0$. This cannot be. Thus all but finitely many $p \in W$ have $f\left(p_{p}\right)=1$. By [4, Theorem 1] all but finitely many $p \in W$ also have height $p=1$. We thus replace $P_{1}$ by some $p_{1} \in W$ with $f\left(p_{p_{p_{1}}}\right)=1=$ height $p_{1}$. The other $p_{i}, i=1,2, \ldots, n-1$, are similarly found.

Let $f$ be a grade function on $R$. In Theorem 4.5 we saw a way of extending $f$ to a grade function $g$ on $R[X]$. If $g$ is locally modulated on $R[X]$, it is trivial to see that $f$ is locally modulated on $R$. For nice rings, the converse also holds, as we will see in Theorem 6.3. (For instance any locally quasi-unmixed ring satisfies the hypothesis of Theorem 6.3, and so fits our present use of 'nice'. Most Noetherian rings found in 
classical situations are locally quasi-unmixed.) However, for nonnice rings the converse may fail.

EXAMPle. By [7, Example 2, pp. 203-205] there is a 2-dimensional local domain $(R, M)$ such that $R[X]$ has primes $0 \subset p \subset q$ with $p \cap R=0$ but $p \neq 0$ and $q \cap R=M$ but $q \neq M R[X]$, and with height $(q / p)=1$. Let $f$ be the height function on $R$ which is locally modulated. Now $g$ is the height function on $R[X]$, but is not locally modulated since height $q=3>$ height $p+1$. Our next result shows that this is the only sort of way that $g$ can escape being locally modulated if $f$ is.

THEOREM 6.3. Let $X$ be an indeterminate over $R$. Suppose whenever $p \subset q$ are primes in $R[X]$ with $p \neq(p \cap R) R[X]$ and $q \neq(q \cap R) R[X]$, if height $q / p=1$ then height $q \cap R / p \cap R=1$. Furthermore, let $f$ be a locally modulated grade function on $R$, and let $g$ be the grade function on $R[X]$ defined in Theorem 4.5. Then $g$ is locally modulated on $R[X]$.

Proof. Let $p \subset q$ be primes of $R[X]$ with height $(q / p)=1$. By Theorem 6.1 we need $f\left(q_{q}\right) \leqslant f\left(p_{p}\right)+1$. We proceed in various ways, using $P=p \cap R$ and $Q=q$ $\cap R$.

Case 1: $p \neq P R[X]$ and $q \neq Q R[X]$. Then by hypothesis, height $(Q / P)=1$, and since $f$ is modulated, $f\left(Q_{Q}\right) \leqslant f\left(P_{P}\right)+1$. By definition of $g, g\left(q_{q}\right)=f\left(Q_{Q}\right)+1 \leqslant$ $f\left(P_{P}\right)+2=g\left(p_{p}\right)+1$.

Case 2: $p=P R[X]$ and $q=Q R[X]$. Clearly height $(Q / P)=1$, and $g\left(q_{q}\right)=$ $f\left(Q_{Q}\right) \leqslant f\left(P_{P}\right)+1=g\left(q_{q}\right)+1$.

Case 3: $p \neq P R[X]$ and $q=Q R[X]$. By [4, Theorem 6] there is a prime $P^{\prime}$ of $R$ with $P \subset P^{\prime} \subset Q$ saturated. Since $f$ is modulated, we have $f\left(Q_{Q}\right) \leqslant f\left(P_{P}\right)+2$. Thus $g\left(q_{q}\right)=f\left(Q_{Q}\right) \leqslant f\left(P_{P}\right)+2=g\left(p_{p}\right)+1$. The only remaining case is easy.

REMARK. The converse of Theorem 6.3 fails. That is, $g$ being locally modulated does not imply that $R[X]$ has the stated property. We again take the ring mentioned in the example prior to Theorem 6.3, but this time we define $f$ by specifying $f\left(P_{P}\right)=$ height $P$ for all $P \in \operatorname{spec} R-\{M\}$, while $f(M)=0$. The $g$ produced on $R[X]$ is easily seen to be locally modulated. However we already have primes $p \subset q$ in $R[X]$ with $p \neq(p \cap R) R[X], q \neq(q \cap R) R[X]$, height $(q / p)=1$, but with height $((q \cap R) /(p \cap R))>1$.

LEMMA 6.4. Let $f$ be a grade function on $R$, and let $I$ be an ideal of $R$. There is $a$ maximal ideal $M$ of $R$ such that $I \subseteq M$ and $f(I)=f\left(I_{M}\right)$.

Proof. By Lemma 1.2(v), there is an $I \subseteq P \in \operatorname{spec} R$ with $f(I)=f\left(P_{P}\right)$. Let $M$ be a maximal ideal containing $P$. Now $f\left(I_{M}\right) \geqslant f(I)=f\left(P_{P}\right) \geqslant f\left(P_{M}\right) \geqslant f\left(I_{M}\right)$, and so equally holds throughout.

Recall that a Noetherian ring $R$ satisfies the first chain condition if every maximal chain of primes (i.e. every saturated chain of primes between a minimal prime and a maximal prime) has length equal to $\operatorname{dim} R$.

THEOREM 6.5. Let $f$ be a grade function on $R$. The following are equivalent.

(a) $f$ restricted to $R_{M}$ is modulated and $f(M)=$ height $M$ for all maximal ideals $M$.

(b) $f(I)=$ height $I$ for all ideals $I$ and $R_{M}$ satisfies the first chain condition for all maximal ideals $M$. 
Proof. (a) $\Rightarrow$ (b) Let (a) hold, and suppose for some $I, f(I) \neq$ height $I$. We will derive a contradiction. We have $f(I)<$ height $I$, and by Lemma 6.4, for some maximal $M$ containing $I, f\left(I_{M}\right)=f(I)<$ height $I \leqslant$ height $I_{M}$. We may assume that $R$ is local at $M$. We may also assume that $I$ is maximal with respect to having $f(I)<$ height $I$. Let $x_{1}, \ldots, x_{n}$ be a maximal $f$-sequence in $I$. Then there is some $I \subseteq P \in A_{f}\left(x_{1}, \ldots, x_{n}\right)$. Now height $P \geqslant$ height $I>f(I)=n=f(P)$, and by the maximality of $I, I=P$ is prime. Since, by assumption, $f(M)=$ height $M, I \neq M$. Pick $a \in M-I$. As $f$ is modulated on $R$, the maximality of $I$ gives height $(I, a)=$ $f(I, a) \leqslant f(I)+1<$ height $I+1$. Thus height $(I, a)=$ height $I$, and since $I$ is prime, $a \in I$. This is our contradiction.

We must show that (a) implies $R_{M}$ satisfies the first chain condition for each maximal ideal $M$. Again we may assume $R$ is local at $M$. Let $p_{0} \subset p_{1} \subset \cdots \subset p_{m-1}$ $\subset M$ be a maximal chain of primes. We need height $M=m$. By [4, Theorem 5] we may assume that height $p_{m-1}=m-1$. Let $P=p_{m-1}$. Now $P \subset M$ is saturated and $m-1=$ height $P=f(P)$ (by the first part of this proof). Pick $b \in M-P$. As $f$ is modulated, $f(P, b) \leqslant f(P)+1=m$. Since $P \subset M$ is saturated, $M$ is the only prime containing $(P, b)$, and so $m \geqslant f(P, b)=f(M)=$ height $M$. Since our original chain of primes shows height $M \geqslant m$, we are done.

(b) $\Rightarrow$ (a) Assuming (b), we must show that for any maximal ideal $M, f$ restricted to $R_{M}$ is modulated. If $P \in \operatorname{spec} R$, (b) implies height $P=f(P) \leqslant f\left(P_{P}\right) \leqslant$ height $P$. That is, $f\left(P_{P}\right)=$ height $P$. From this it easily follows that $f$ restricted to $R_{M}$ is just the height function on $R_{M}$. Therefore we may assume that $R$ is local at $M$. Let $I$ be an ideal and $a \in M$. Since $R$ satisfies the first chain condition, $f(I, a)=\operatorname{height}(I, a)$ $\leqslant$ height $I+1=f(I)+1$, so that $f$ is modulated.

REMARKS. (i) It is easily seen that condition (b) in Theorem 6.5 implies that $f$ is locally modulated, since condition (b) localizes.

(ii) We previously noted that if $f$ is any of classical, essential, or asymptotic grade, then $f$ is locally modulated. Thus if on some $\operatorname{ring} R, f(M)=$ height $M$ for all maximal ideals $M$, it follows that $f(I)=$ height $I$ for all ideals $I$.

We now look at unmixedness. The classical unmixedness theorem states that if $R$ is a Cohen-Macaulay ring and $I=\left(x_{1}, \ldots, x_{m}\right)$ with $m=$ height $I$, and $n \geqslant 1$ is an integer, then $P \in \operatorname{Ass}\left(R / I^{n}\right)=A_{1}\left(I^{n}\right)$ implies height $P=$ height $I$. We will show that if the grade function $f$ on $R$ satisfies the equivalent conditions of Theorem 6.5, then with $I$ as above and $A$ any grade scheme for $f, P \in A(I)$ implies height $P=$ height $I$. As for powers of $I$, we show that $P \in A_{f}\left(I^{n}\right)$ implies height $P=$ height $I$, and that this also works for our natural grade schemes $A_{1}$ through $A_{6}$.

THEOREM 6.6 Let $f$ be a grade function on $R$ and suppose for each maximal ideal $M$ of $R, f(M)=$ height $M$ and $f$ is modulated on $R_{M}$. Let $A$ be any grade scheme for $f$, and let $I=\left(x_{1}, \ldots, x_{m}\right)$ with $m=$ height $I$. Also let $n \geqslant 1$ be an integer.

(a) If $P \in A(I)$, then height $P=$ height $I$.

(b) If $A \subseteq A_{f}$ and if $P \in A\left(I^{n}\right)$, then height $P=$ height $I$.

(c) If $f$ is classical grade and either $P \in A_{1}\left(I^{n}\right)$ or $P \in A_{2}\left(I^{n}\right)$, then height $P=$ height $I$. 
(d) If $f$ is essential grade and either $P^{\oplus} \in A_{3}\left(I^{n}\right)$ or $P \in A_{4}\left(I^{n}\right)$, then height $P=$ height $I$.

(e) If $f$ is asymptotic grade and either $P \in A_{5}\left(I^{n}\right)$ or $P \in A_{6}\left(I^{n}\right)$, then height $P=$ height $I$.

Proof. (a) Since condition (a) of Theorem 6.5 holds, so does condition (b). Thus $f$ is just the height function (on all localizations of $R$ by the previous remark). We consider the case $A=A_{f}$. If $P \in A_{f}(I)$ then height $P=f\left(P_{P}\right)=f\left(I_{P}\right)=$ height $I_{P}$ $=$ height $I$, the last equality following from the hypothesis on $I$. Thus (a) holds when $A=A_{f}$. For arbitrary $A$, if $P \in A(I)$ then since the hypotheses localize, we may assume that $R$ is local at $P$ and satisfies the first chain condition (Theorem 6.5(b)). Since $I=\left(x_{1}, \ldots, x_{m}\right)$ with $m=$ height $I$, it is not hard to see that height $\left(x_{1}, \ldots, x_{i}\right)$ $=i$ for all $i=1, \ldots, m$, and so by the first part of this proof, $A_{f}\left(x_{1}, \ldots, x_{i}\right)$ consists of the primes minimal over $\left(x_{1}, \ldots, x_{i}\right)$. Therefore we see that $x_{1}, \ldots, x_{m}$ is an $f$-sequence. Using Lemma 1.4, $P \in A(I)=A_{f}(I)$ and so height $P=$ height $I$ by the preceding.

(b) Since $f$ is just height, $A_{f}(J)$ consists of the primes minimal over $J$ for any ideal $J$. Thus $A \subseteq A_{f}$ implies $A=A_{f}$, and also $A_{f}\left(I^{n}\right)=A_{f}(I)$. Therefore $P \in A\left(I^{n}\right)$ implies $P \in A_{f}(I)$ and so height $P=$ height $I$.

(c) In part (a) we saw that $x_{1}, \ldots, x_{m}$ is an $f$-sequence. By [1, Exercise 13, p. 103], $A_{1}\left(I^{n}\right)=A_{1}(I)$. Thus $P \in A_{1}\left(I^{n}\right)$ implies height $P=$ height $I$ by part (a). Now for any ideal $J$, it is easily seen that $A_{2}\left(J^{n}\right)=A_{2}(J)$, and so the same argument works for $A_{2}$.

(d) For any ideal $J$ it is easily seen that $A_{3}\left(J^{n}\right)=A_{3}(J)$ and $A_{4}\left(J^{n}\right)=A_{4}(J)$. Thus argue as in (c).

(e) For any ideal $J, A_{5}\left(J^{n}\right)=A_{5}(J)$ and $A_{6}\left(J^{n}\right)=A_{6}(J)$. The latter is easy. The former uses [5, Propositions 3.18 and 4.1]. Now argue as in (c).

Finally, a difficult question.

Question. Let $R$ and $R^{\prime}$ be two Noetherian rings such that $\operatorname{spec} R$ and $\operatorname{spec} R^{\prime}$ are isomorphic as partially ordered sets under inclusion. Then a grade function $f$ on $R$ can be transferred to $R^{\prime}$ by letting $f^{\prime}\left(P_{P^{\prime}}^{\prime}\right)=f\left(P_{P}\right)$, where $P^{\prime} \in \operatorname{spec} R^{\prime}$ is the image of $P \in \operatorname{spec} R$ under the isomorphism. Suppose that $f$ is a locally modulated grade function on $R$. Is there an $R^{\prime}$ with $\operatorname{spec} R^{\prime} \approx \operatorname{spec} R$ such that the transferred grade function $f^{\prime}$ on $R^{\prime}$ equals classical grade (or essential or asymptotic grade) on $R^{\prime}$ ?

\section{Sequences over ideals.}

Definition. Let $A$ be a grade scheme on $R$, and let $I$ be an ideal of $R$. Define an $A$-sequence over $I$ to be a sequence $x_{1}, \ldots, x_{n}$ of elements of $R$ such that $\left(I, x_{1}, \ldots, x_{n}\right) \neq R$ and for each $i=1, \ldots, n, x_{i} \notin A\left(I, x_{1}, \ldots, x_{i-1}\right)$.

In [5, Chapter VI] this concept is considered for $A_{5}$, while [3 and 11] look at it for $A_{3}$ and $A_{4}$. (To the best of our knowledge it has not been studied for $A_{1}, A_{2}$ or $A_{6}$.) We note that since $A_{4} \subseteq A_{3}$ an $A_{3}$-sequence over $I$ will be an $A_{4}$-sequence over $I$, but the converse fails despite the fact that $A_{3}$ and $A_{4}$ are both grade schemes for essential 
grade. Thus we must specify the grade scheme, not just the grade function. Also it is not hard to find a grade scheme on a local ring and an ideal $I$ having two maximal $A$-sequences over $I$ of different length. ( $A_{3}, A_{4}$ and $A_{5}$ escape this flaw.)

In this section we will look at $A$-sequences over $I$ when $A$ is a grade scheme for $f$ and $A_{f} \subseteq A$. We give a very easy proof of a generalization of the following known facts. If $f$ is essential (respectively, asymptotic) grade, $A$ is $A_{3}$ (respectively, $A_{5}$ ) and $I$ is an ideal in a local ring with $x_{1}, \ldots, x_{n}$ and $A$-sequence over $I$, then $n \leqslant f(M)-f(I)$ [3, Theorem 6.5] (respectively, [5, Proposition 6.9]). We then consider $A_{f}$-sequences over $I$ when $f$ is locally modulated.

LEMMA 7.1. Let $f$ be a grade function on $R$. Let $A$ be any grade scheme for $f$ with $A_{f} \subseteq A$. Let $I$ be an ideal and let $x_{1}, \ldots, x_{n}$ be an $A$-sequence over I. Then $f\left(I, x_{1}, \ldots, x_{n}\right) \geqslant f(I)+n$.

Proof. Since $x_{2}, \ldots, x_{n}$ is an $A$-sequence over $\left(I, x_{1}\right)$, it will suffice to let $n=1$. Clearly $f\left(I, x_{1}\right) \geqslant f(I)$. If equality holds, then for some $\left(I, x_{1}\right) \subseteq P \in \operatorname{spec} R$, $f\left(P_{P}\right)=f\left(I, x_{1}\right)=f(I)$. However, this shows that $P \in A_{f}(I) \subseteq A(I)$, contradicting that $x_{1}$ is an $A$-sequence over $I$.

Since $A_{f} \subseteq A_{4} \subseteq A_{3}$ for $f=$ essential grade, and $A_{f} \subseteq A_{6} \subseteq A_{5}$ for $f=$ asymptotic grade, our next theorem incorporates the known results mentioned above.

THEOREM 7.2. Let $f$ be a grade function on a local ring $(R, M)$ and let $A$ be any grade scheme for $f$ with $A_{f} \subseteq A$. If $x_{1}, \ldots, x_{n}$ is an A-sequence over I, then $n \leqslant f(M)$ $-f(I)$.

Proof. $f(M) \geqslant f\left(I, x_{1}, \ldots, x_{n}\right) \geqslant f(I)+n$ by Lemma 7.1.

LEMMA 7.3. Let $f$ be a modulated grade function on a local ring $(R, M)$, and let $A$ be any grade scheme for $f$ with $A_{f} \subseteq A$. Let $I$ be an ideal and let $x_{1}, \ldots, x_{n}$ be an $A$-sequence over $I$. Then $f\left(I, x_{1}, \ldots, x_{n}\right)=f(I)+n$.

Proof. Lemma 7.1 gives one inequality and modulation gives the other.

REMARK. Lemma 7.3 requires that $R$ be local, even if $f$ is locally modulated.

THEOREM 7.4. Let $f$ be modulated on a local ring $(R, M)$. Let $I$ be an ideal, and let $x_{1}, \ldots, x_{n}$ be a maximal $A_{f}$-sequence over $I$. Then $n=f(M)-f(I)$.

Proof. By maximality, $M \in A_{f}\left(I, x_{1}, \ldots, x_{n}\right)$. Thus $f(M)=f\left(I, x_{1}, \ldots, x_{n}\right)=$ $f(I)+n$, using Lemma 7.3.

The next corollary is similar to [5, Proposition 6.14 and 3, Theorem 6.2].

COROLlaRy 7.5. Let $f$ be a locally modulated grade function on a local ring $(R, M)$. Let $A$ be any grade scheme for $f$ with $A_{f} \subseteq A$. Let $I$ be an ideal and let $x_{1}, \ldots, x_{n}$ be an $A$-sequence over $I$. Then $n \leqslant \min \left\{\right.$ little depth $P \mid I \subseteq P \in \operatorname{spec} R$ and $\left.f(I)=f\left(P_{P}\right)\right\}$.

Proof. We have $n \leqslant f(M)-f(I)$ by Theorem 7.2. If $P$ is as above, then $n \leqslant f(M)-f\left(P_{P}\right)$. Suppose little depth $P=m$. Then Theorem 6.1 easily shows $f(M) \leqslant f\left(P_{P}\right)+m$, and so $n \leqslant m$. 
COROLlaRY 7.6. Let $f$ be a locally modulated grade function on $R$. Let I be an ideal and let $x_{1}, \ldots, x_{n}$ be an $A_{f}$-sequence over $I$. Let $I \subseteq P \in \operatorname{spec} R$ with $f(I)=f\left(P_{P}\right)$. If $\left(P, x_{1}, \ldots, x_{n}\right) \neq R$, then $x_{1}, \ldots, x_{n}$ is an $A_{f}$-sequence over $P$.

Proof. We induct on $n$, the case $n=0$ being trivial. Thus suppose we already have that $x_{1}, \ldots, x_{n-1}$ is an $A_{f}$-sequence over $P$. If our result fails, then for some $Q \in A_{f}\left(P, x_{1}, \ldots, x_{n-1}\right)$ we have $x_{n} \in Q$. It is clear that in $R_{Q}, x_{1}, \ldots, x_{n}$ and $x_{1}, \ldots, x_{n-1}$ are $A_{f}$-sequences over $I_{Q}$ and $P_{Q}$ respectively, the latter being maximal. By Theorem 7.4, $f\left(Q_{Q}\right)-f\left(P_{Q}\right)=n-1$ and $f\left(Q_{Q}\right)-f\left(I_{Q}\right) \geqslant n$. Therefore $f\left(P_{Q}\right)$ $>f\left(I_{Q}\right)$. However, by hypothesis, $f\left(P_{P}\right)=f(I) \leqslant f\left(I_{Q}\right)<f\left(P_{Q}\right) \leqslant f\left(P_{P}\right)$, an obvious contradiction.

EXAMPLE. The preceding fails for arbitrary $P \in A_{f}(I)$. Let $(R, M)$ be a 3-dimensional local domain. Let $Q$ and $P$ be height 2 primes and let $I=P \cap Q$. Define $f$ by specifying $f\left(p_{p}\right)=$ height $p$ for all $p \in \operatorname{spec} R-\{Q, M\}$, while $f\left(Q_{Q}\right)=1$ and $f(M)=2$. Thus $f$ is locally modulated. Clearly $A_{f}(I)=\{Q, P\}$. Pick $x \in M-(Q$ $\cup P$ ) so that $x$ is an $A_{f}$-sequence over $I$. However $x$ is not an $A_{f}$-sequence over $P$ since $f(M)=f(P)$ implies $M \in A_{f}(P)$.

Proposition 7.7. Let $f$ be a locally modulated grade function on $R$. Let I be an ideal and let $x_{1}, \ldots, x_{n}$ be an $A_{f}$-sequence over $I$. Suppose $Q$ is a prime containing $\left(I, x_{1}, \ldots, x_{n}\right)$. The following are equivalent.

(i) $Q \in A_{f}\left(I, x_{1}, \ldots, x_{n}\right)$.

(ii) $f\left(Q_{Q}\right)=f\left(I_{Q}\right)+n$.

(iii) There is a $P \in A_{f}(I)$ with $P \subseteq Q, f\left(P_{P}\right)=f\left(I_{Q}\right)$ and $Q \in A_{f}\left(P, x_{1}, \ldots, x_{n}\right)$.

Proof. (i) $\Rightarrow$ (ii). If (i) holds, then in $R_{Q}, x_{1}, \ldots, x_{n}$ is a maximal $A_{f}$-sequence over $I_{Q}$, and so Theorem 7.4 gives (ii).

(ii) $\Rightarrow$ (iii). Suppose (ii) holds. There is some $I_{Q} \subseteq P_{Q} \in \operatorname{spec} R_{Q}$ with $f\left(I_{Q}\right)=$ $f\left(P_{P}\right)$. Clearly $P \in A_{f}(I)$. By Corollary 7.6 applied in $R_{Q}, x_{1}, \ldots, x_{n}$ is an $A_{f^{-}}$ sequence over $P_{Q}$. By Lemma 7.3, $f\left(\left(P, x_{1}, \ldots, x_{n}\right) R_{Q}\right)=f\left(P_{Q}\right)+n$. Since $f\left(P_{P}\right)=$ $f\left(I_{Q}\right) \leqslant f\left(P_{Q}\right) \leqslant f\left(P_{P}\right)$, we have $f\left(P_{Q}\right)=f\left(I_{Q}\right)$. Thus $f\left(\left(P, x_{1}, \ldots, x_{n}\right) R_{Q}\right)=f\left(I_{Q}\right)$

$+n=f\left(Q_{Q}\right)$ by (ii). This shows $Q \in A_{f}\left(P, x_{1}, \ldots, x_{n}\right)$.

(iii) $\Rightarrow$ (i). If (iii) holds, then

$$
\begin{aligned}
f\left(Q_{Q}\right) & =f\left(\left(P, x_{1}, \ldots, x_{n}\right) R_{Q}\right) \leqslant f\left(P_{Q}\right)+n \quad \text { (by local modulation) } \\
& \leqslant f\left(P_{P}\right)+n=f\left(I_{Q}\right)+n \leqslant f\left(\left(I, x_{1}, \ldots, x_{n}\right) R_{Q}\right) \quad \text { (by Lemma 7.1) } \\
& \leqslant f\left(Q_{Q}\right) .
\end{aligned}
$$

Equality holds throughout, and so $Q \in A_{f}\left(I, x_{1}, \ldots, x_{n}\right)$.

COROllary 7.8. Let $f$ be a locally modulated grade function on $R$. Let I be an ideal. Suppose $x_{1}, \ldots, x_{n}$ is an $A_{f}$-sequence over $P$ for every $P \in A_{f}(I)$. Then $x_{1}, \ldots, x_{n}$ is an $A_{f}$-sequence over $I$.

Proof. We induct on $n$. Suppose we already know $x_{1}, \ldots, x_{n-1}$ is an $A_{f}$-sequence over $I$. If our result fails, then for some $Q \in A_{f}\left(\mathrm{I}, x_{1}, \ldots, x_{n-1}\right)$ we have $x_{n} \in Q$. By Proposition 7.7, for some $P \in A_{f}(I), Q \in A_{f}\left(P, x_{1}, \ldots, x_{n-1}\right)$. Since $x_{n} \in Q$ we have contradicted that $x_{1}, \ldots, x_{n}$ is an $A_{f}$-sequence over $P$. 
Remark. Comparing Corollary 7.6 (and the example following it) to Corollary 7.8 shows that it might be of interest to study the set of ideals $I$ for which $A_{f}(I)=\{P$ $\in \operatorname{spec} R \mid I \subseteq P$ and $\left.f(I)=f\left(P_{P}\right)\right\}$. We will resist the temptation to do so here, except to point out that $I$ has this property if and only if $f\left(P_{P}\right)=f(I)$ for every minimal prime divisor $P$ of $I$, as is easily seen.

\section{BIBLIOGRAPHY}

1. I. Kaplansky, Commutative rings, Univ. of Chicago Press, Chicago, Ill., 1974.

2. D. Katz, Prime divisors, asymptotic R-sequences, and unmixed local rings (manuscript).

3. D. Katz and L. J. Ratliff, Jr., Essential sequences over an ideal and essential cograde. II (manuscript). 4. S. McAdam, Saturated chains in Noetherian rings, Indiana Univ. Math. J. 23 (1974), 719-728.

5. New York, 1983. Asymptotic prime divisors, Lecture Notes in Math., vol. 1023. Springer-Verlag, Berlin and

6. S. McAdam and L. J. Ratliff, Jr., Essential sequences (manuscript).

7. M. Nagata, Local rings, Interscience, New York, 1962.

8. L. J. Ratliff, Jr., Characterizations of catenary rings, Amer. J. Math. 93 (1971), 1070-1108.

9. On asymptotic prime divisors (manuscript).

10. __ Asymptotic sequences, J. Algebra 85 (1983), 337-360.

11. Essential sequences over ideals and essential cograde (manuscript).

Department of Mathematics, University of Texas, Austin, Texas 78712 